\title{
Tissue-specific variation in DNA methylation levels along human chromosome I
}

\author{
Cecilia De Bustos ${ }^{\dagger 1,8}$, Edward Ramos ${ }^{\dagger 2,3,9}$, Janet M Young ${ }^{\dagger 2}$, \\ Robert K Tran ${ }^{4,10}$, Uwe Menzel1 ${ }^{1}$, Cordelia F Langford ${ }^{5}$, Evan E Eichler ${ }^{3,6}$, \\ $\mathrm{Li} \mathrm{Hsu}^{7}$, Steve Henikoff4,6, Jan P Dumanski ${ }^{1}$ and Barbara J Trask*2,3
}

\begin{abstract}
Address: ${ }^{1}$ Department of Genetics and Pathology, Rudbeck Laboratory, Uppsala University, Uppsala, Sweden, ${ }^{2}$ Division of Human Biology, Fred Hutchinson Cancer Research Center, Seattle, Washington, USA, ${ }^{3}$ Department of Genome Sciences, University of Washington, Seattle, Washington, USA, ${ }^{4}$ Division of Basic Sciences, Fred Hutchinson Cancer Research Center, Seattle, Washington, USA, ${ }^{5}$ The Wellcome Trust Sanger Institute, Wellcome Trust Genome Campus, Hinxton, Cambridge, UK, ${ }^{6}$ Howard Hughes Medical Institute, Seattle, Washington, USA, ${ }^{7}$ Division of Public Health Sciences, Fred Hutchinson Cancer Research Center, Seattle, Washington, USA, ${ }^{8}$ Current address: United Nations World Food Programme, Lima, Peru, ${ }^{9}$ Current address: National Institutes of Health, Bethesda Maryland, USA and ${ }^{10}$ Current address: Genome Center, University of California at Davis, Davis, California, USA

Email: Cecilia De Bustos - cdebustos@gmail.com; Edward Ramos - ramose@mail.nih.gov; Janet M Young - jayoung@fhcrc.org; Robert KTran - rktran@ucdavis.edu; Uwe Menzel - uwe.menzel@genpat.uu.se; Cordelia F Langford - cfl@sanger.ac.uk; Evan E Eichler - eee@gs.washington.edu; Li Hsu - lih@fhcrc.org; Steve Henikoff - steveh@fhcrc.org; Jan P Dumanski - jan.dumanski@genpat.uu.se; Barbara J Trask* - btrask@fhcrc.org

* Corresponding author †Equal contributors
\end{abstract}

Published: 8 June 2009

Epigenetics \& Chromatin 2009, 2:7 doi:10.1186/1756-8935-2-7
Received: 8 December 2008

Accepted: 8 June 2009

This article is available from: http://www.epigeneticsandchromatin.com/content/2/I/7

(C) 2009 De Bustos et al; licensee BioMed Central Ltd.

This is an Open Access article distributed under the terms of the Creative Commons Attribution License (http://creativecommons.org/licenses/by/2.0), which permits unrestricted use, distribution, and reproduction in any medium, provided the original work is properly cited.

\begin{abstract}
Background: DNA methylation is a major epigenetic modification important for regulating gene expression and suppressing spurious transcription. Most methods to scan the genome in different tissues for differentially methylated sites have focused on the methylation of $C_{p} G$ s in $C_{p} G$ islands, which are concentrations of $C_{P G}$ s often associated with gene promoters.
\end{abstract}

Results: Here, we use a methylation profiling strategy that is predominantly responsive to methylation differences outside of $C_{P G}$ islands. The method compares the yield from two samples of size-selected fragments generated by a methylation-sensitive restriction enzyme. We then profile nine different normal tissues from two human donors relative to spleen using a custom array of genomic clones covering the euchromatic portion of human chromosome I and representing $8 \%$ of the human genome. We observe gross regional differences in methylation states across chromosome I between tissues from the same individual, with the most striking differences detected in the comparison of cerebellum and spleen. Profiles of the same tissue from different donors are strikingly similar, as are the profiles of different lobes of the brain. Comparing our results with published gene expression levels, we find that clones exhibiting extreme ratios reflecting low relative methylation are statistically enriched for genes with high expression ratios, and vice versa, in most pairs of tissues examined.

Conclusion: The varied patterns of methylation differences detected between tissues by our methylation profiling method reinforce the potential functional significance of regional differences in methylation levels outside of $C_{P G}$ islands. 


\section{Background}

DNA methylation is a major epigenetic modification that is vital to mammalian development [1]. Methylation is carried out by DNA methyltransferases [2] and can suppress the initiation of transcription of a locus [3]. Methylation plays a significant role in genomic imprinting, $\mathrm{X}$ inactivation, and silencing of parasitic sequences [4-7]. Acquired DNA methylation differences might account for some phenotypic differences between monozygotic twins [8]. Aberrant methylation can cause various syndromes [9-14] and contribute to tumorigenesis by decreasing activity of tumor suppressor genes [15], activating protooncogenes [16], or overall methylation imbalance [17] (reviewed in [18]).

In mammals, methylation occurs preferentially at cytosines that are followed by guanine (CpG). CpG dinucleotides are relatively infrequent in the human genome, except in CpG islands, which are small (0.2 to $2 \mathrm{~kb})$ regions highly enriched in CpGs. Approximately 50 to $60 \%$ of gene promoters lie in CpG islands [19-21]. Methylation can inhibit binding of transcription factors to these sequences directly by altering the structure of the recognition site, or indirectly by recruiting repressive proteins with methyl-binding domains [22-25].

However, methylation of CpG island promoters does not always correlate with gene expression levels. Most inactive promoters are not methylated $[3,26]$. The CpG islands of some tissue-specific genes remain unmethylated in tissues where the gene is not expressed (such as MyoD in nonmuscle tissues) [27-29]. Intriguingly, while promoters are less methylated on the active $\mathrm{X}$ than on the inactive $\mathrm{X}$, the opposite is true for CpGs in the bodies of genes on the $\mathrm{X}$ chromosome [30]. Highly expressed autosomal genes were also found to have hypomethylated promoters and hypermethylated gene bodies in a recent genome-wide analysis [31]. Moreover, clusters of hypomethylated regions not limited to $\mathrm{CpG}$ islands or promoters have been found interspersed in stretches of methylated CpGs [32]. CpG methylation outside of CpG islands is thought to suppress transcription of transposable elements and other parasitic sequences [26,33] and/or to suppress spurious initiation of transcription elsewhere, including within infrequently transcribed genes [34,35]. Clearly more information on the state of methylation in and outside of $\mathrm{CpG}$ islands and across different tissues is needed to clarify the role methylation plays in transcriptional regulation.

Several approaches have been developed recently to scan genomes for sites of DNA methylation that might be important for tissue-specific gene regulation. These approaches include large-scale sampling of DNA sequences after bisulfite conversion of unmethylated cytosines to uracil $[31,33,36-38]$, a variety of techniques measuring the differential sensitivity of methylated and unmethylated sequences to certain restriction enzymes (for example, RLGS, RDA, HELP, MCAM, and MSCC) $[31,32,39-48]$, and quantitative analyses of promoter sequences immunoprecipitated with antibodies that enrich for methylated cytosines $[26,49,50]$ (reviewed in [51]). Differences in methylation have been detected among somatic tissues at up to $15 \%$ of the loci analyzed in these studies $[26,31,33,36,45,48,52]$.

Here, we employ a methylation profiling technique and a tiling microarray for human chromosome 1 to detect regional differences in the methylation state between tissues from the same individual. Our approach relies on a methylation-sensitive restriction enzyme to generate differently sized fragments from methylated versus nonmethylated genomic DNA. Sample differences in fragment yield within a specified size range ( 80 to $2,500 \mathrm{bp}$ ) are detected by competitive hybridization of size-selected fragments to a tiling array of genomic clones. This approach has been used successfully to characterize methylation patterns in Arabidopsis methylation mutants $[53,54]$. We have combined this technique with a custom chromosome 1 microarray with a resolution of approximately $100 \mathrm{~kb}$ [55].

Below, we first describe simulation experiments that show that $\log$ ratios measured by the array are a complex function of the methylation level of the hybridizing DNA and the GC-content of the array clones. We also predict from simulations that the array will be more responsive to methylation changes at dispersed $\mathrm{CpG}$ sites than to changes in $\mathrm{CpG}$ islands. We then use the array to profile chromosome 1 methylation in several tissues from two human donors, demonstrating that the technique yields highly reproducible results. The methylation profiles we obtain vary between different human tissues, but profiles of the same tissue from two different individuals are similar. We then describe our use of bisulfite sequencing to confirm an array finding of hypomethylation in heart DNA around the RYR2 gene and to confirm predictions from our simulations that observed log ratios are impacted by methylation differences outside of CpG islands. We analyze published data on the relative expression levels of genes and detect significantly different expression ratios for genes residing in regions exhibiting extremely high versus low methylation ratios in all six of the tissue comparisons analyzed.

\section{Results \\ Simulations predict impact of GC content and CPG distribution on fragment yield}

In our methylation-profiling technique (Figure 1), DNAs obtained from test and reference samples are digested with a methylation-sensitive restriction enzyme, HpaII, which cleaves CCGG sites that are not modified by meth- 
(1)

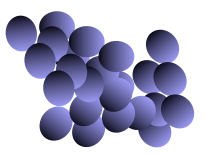

Harvest DNA from test \& reference samples

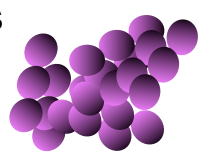

(2) Digest each to completion with methylation-sensitive Hpall
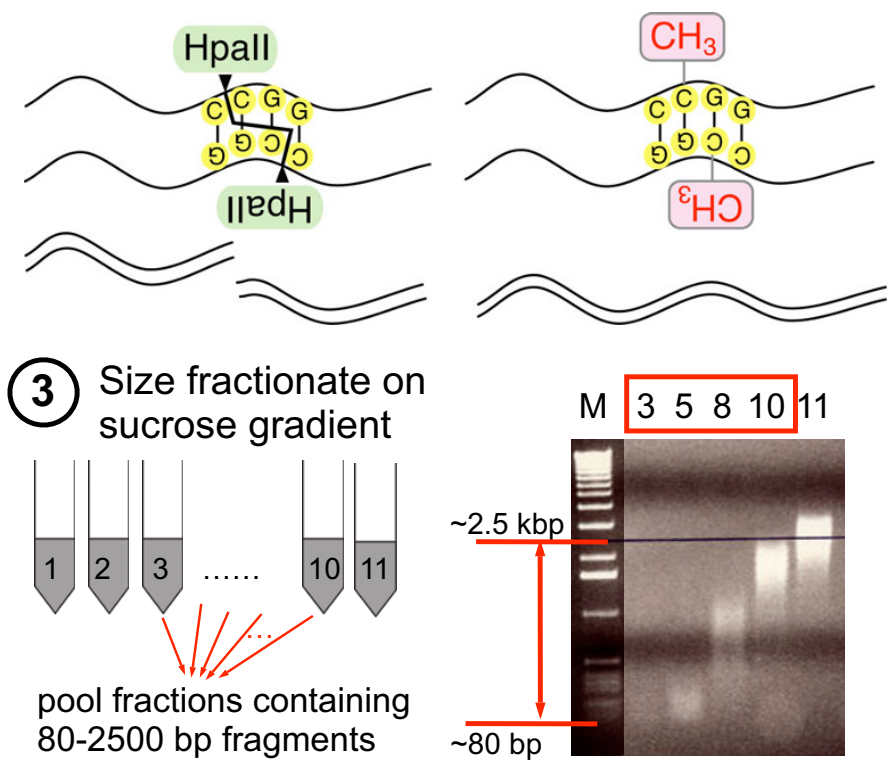

pool fractions containing $80-2500 \mathrm{bp}$ fragments

$\sim 80 \mathrm{bp}$
(4) Differentially label test and reference

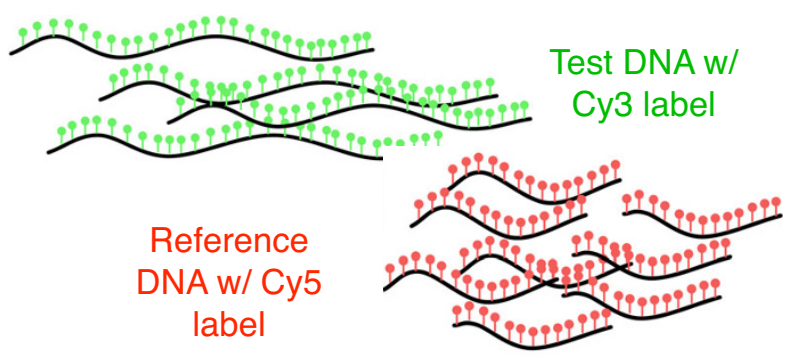

(5) Competitively hybridize, suppressing repeats, to array of $\sim 2100$ large-insert clones covering euchromatic portions of chromosome 1

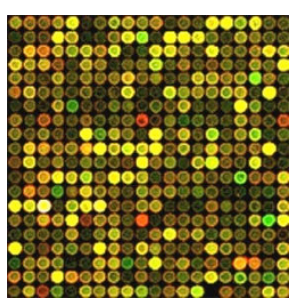

(6) Analyze relative fluorescence intensities

\section{Figure I}

Schematic of methylation profiling technique. DNA is harvested from samples (I) and digested with methylation-sensitive enzyme, Hpall, which cuts at its CCGG target site only if the CpG is not methylated (2). The DNA is then size-fractionated on a sucrose gradient, followed by determination of fragment size by agarose gel electrophoresis in order to choose fractions containing fragments > approximately $80 \mathrm{bp}$ and < approximately $2.5 \mathrm{~kb}$; in this example, fractions represented by lanes numbered 3 to 10 (3). Test and reference samples are then differentially labeled (4) and competitively hybridized to a clone-based microarray containing 2,049 clones for chromosome I, 17 clones for the X chromosome, and 70 other clones (5). The signal intensities of each fluorescence channel are expressed as a ratio, $\log _{2}$ transformed, and then loess normalized on the percent GC content of clone sequences (6).

ylation. Therefore, the methylation status for each sample influences the size distribution of restriction fragments: unmethylated DNA is cut into smaller pieces than is highly methylated DNA. After digestion, fragments longer than approximately $80 \mathrm{bp}$ and shorter than approximately $2.5 \mathrm{~kb}$ are isolated by sucrose-gradient fractionation, and test and reference DNAs are differentially labeled with fluorochromes by random priming. Thus, size fractionation is the primary determinant for distinguishing between two differentially methylated samples.

These samples are allowed to competitively hybridize to 2,136 genomic clones (bacterial artificial chromosomes (BACs), P1-derived artificial chromosomes (PACs), fosmids, cosmids) on a microarray in the presence of Cot 1
DNA to suppress repetitive sequences. The array contains 2,049 clones that cover most of the euchromatic portions of human chromosome 1, 17 clones that are distributed sparsely on chromosome $\mathrm{X}$, and 70 other clones. Average $\log _{2}$ ratios of test-to-reference fluorescence intensities are generated from the duplicate spots for each clone whose measurements pass quality control tests (see Methods). Since the median size of the genomic inserts of the clones on the array is $159 \mathrm{~kb}$, the signal ratio for a given clone is a complex function of the methylation states of HpaII sites in the sample and reference (which might both be heterogeneous mixtures of cells with different methylation patterns) and the cumulative length (minus repeats) of the resulting HpaII fragments within the size range of approx- 
imately 80 to 2,500 bp in the genomic interval covered by the clone.

We used simulations to understand how methylation differences between samples might influence the relative fluorescence ratios measured for large-insert clones on the array. In order for the amount of hybridizing material generated from two samples to differ, the methylation state of the sample must yield, after HpaII digestion, a significantly higher or lower amount of material in fragments within the size range 80 to 2,500 bp from the test sample compared with the reference. Methylation can impact the amount of product in two opposing ways (Figure 2A). For example, consider two neighboring HpaII fragments, each around $2 \mathrm{~kb}$ in size. If the HpaII site between them is methylated, then the fragments remain joined and exceed the upper size cut-off ( $2.5 \mathrm{~kb})$. Here, methylation reduces fragment yield through the size filter (fragments adjoining sites marked "a" in Figure 2A). Conversely, methylation could increase fragment yield through the size filter for other regions, such as CpG islands, which have a high density of HpaII sites (fragments adjoining sites marked " $\mathrm{c}$ " in Figure 2A). HpaII fragments derived from unmethylated CpG islands could be excluded due to their small size (<80 bp), whereas HpaII's inability to cut the same regions when methylated could generate fragments that pass the size filter. In yet other cases, methylation might have no effect on the amount of size-selected hybridizing material (sites marked "b" in Figure 2A).

In order to simulate the relative fragment yield for an entire BAC at different methylation levels, we wrote a PERL script to randomly assign each HpaII site in a BAC's sequence to be methylated or unmethylated, so as to achieve various user-specified average overall levels of methylation. The script performed in silico HpaII digestion of the resulting partially methylated sequence and the size-fractionation step. It also masked the sequences using RepeatMasker [56], which mimics the Cot1 repeat-suppression step necessary to achieve specificity in array hybridization. The output is total unmasked base pairs in fragments passing the size filter, which simulates labeling by random priming [57].

Our simulations show that yield of total unmasked base pairs passing the size filter from the region corresponding to any given clone on the array decreases approximately linearly with increasing average per cent methylation. Figure $2 \mathrm{~B}$ shows the results across a range of methylation levels for BAC RP11-47A4 (AL391809), an outlying clone in the heart-versus-spleen comparisons and the object of experimental validation below. This negative slope indicates that the array response is dominated by methylation status at HpaII sites between larger fragments where increased methylation would reduce yield through the size filter (sites of type a in Figure 2A), and that smaller HpaII fragments like those in CpG islands (sites of type c in Figure 2A) have a negligible effect on the yield of sizeselected fragments corresponding to any array clone. Simulations for all clones on the array show a similar negative correlation between fragment yield through the size filter and methylation level. Figure $2 \mathrm{C}$ and 2D display results for two selected methylation levels, demonstrating that a sample uniformly methylated at $40 \%$ of sites (Figure 2C, black contours) yields more hybridizing material for all array BACs than a sample with $80 \%$ methylation (Figure $2 \mathrm{C}$, gray contours) and positive predicted log ratios for all array BACs (Figure 2D). A caveat of our simulations is that we assumed that all $\mathrm{CpG}$ dinucleotides had an equal chance of being methylated. More sophisticated simulations might model local heterogeneity, such as that observed in our bisulfite sequencing analyses (see Results), or the differences between CpG islands and other regions, but would need a good model to describe how methylation is distributed across sites. Additional simulations show that the relationship between fragment yield and methylation level would have a positive slope only if CpG islands comprise more than $30 \%$ of a clone's sequence (data not shown), and that is not the case for any clone on the array. Thus, signal intensity differences between clones or samples are likely to be predominated by methylation differences at CpGs outside of CpG islands.

Simulations also demonstrate a strong relationship between fragment yield and the percentage of bases that are either $\mathrm{G}$ or $\mathrm{C}(\mathrm{GC} \%)$ in the probed sequence. Figure 2A helps explain how GC content can affect measured $\log _{2}$ ratios. In this schematic example, HpaII sites are relatively frequent in BAC 1 , so that many fragments pass the size filter in both samples 1 and 2. The increased methylation of sample 2 causes a relatively modest reduction in hybridizing material for BAC 1 . In contrast, BAC 2 has lower GC content and thus larger HpaII fragments, on average, and therefore the same increased methylation level in sample 2 has a much more dramatic effect, entirely preventing any material passing the 80 to 2,500 bp size filter. This more dramatic effect would result in a more extreme measured log ratio for BAC 2 than BAC 1 despite the fact that both BACs measure the same pair of samples with the same ratio of methylation levels. Figure $2 \mathrm{C}$ shows the positive relationship between total base pairs in HpaII fragments predicted to pass our size filter and GC\% for the regions represented by the clones on our array. This positive relationship is seen at all simulated methylation levels $(40 \%$ and $80 \%$ are shown in Figure 2C), except at the extreme of $100 \%$ methylation, when the DNA would not be cut at all. Moreover, the $\log _{2}$ ratio of yield for two samples simulated to have different overall methylation levels (test low, reference high) is positive for 
A

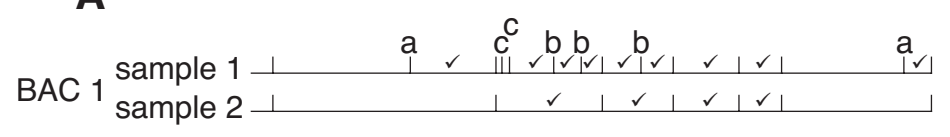

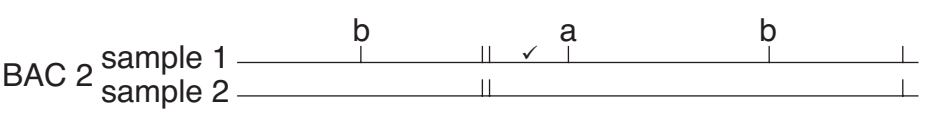
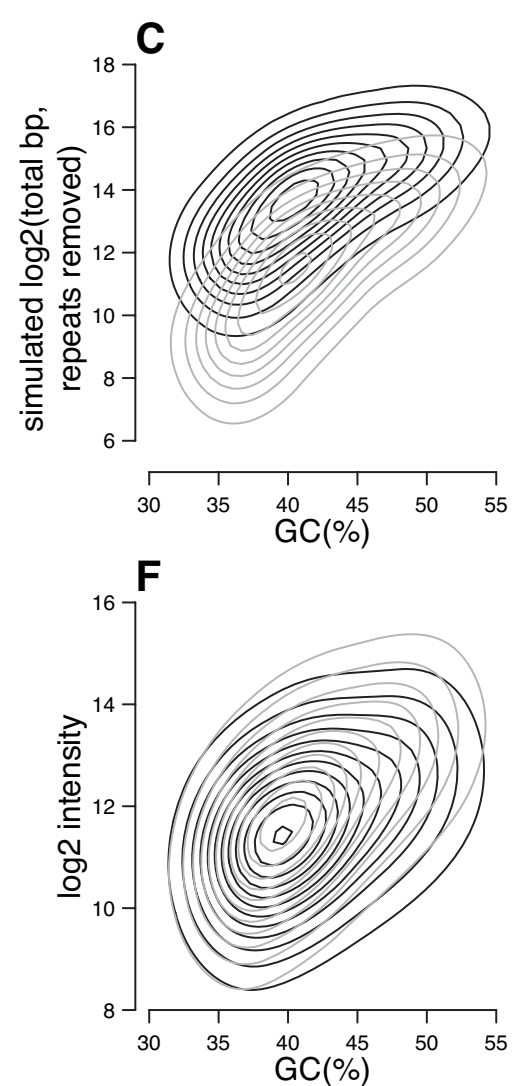

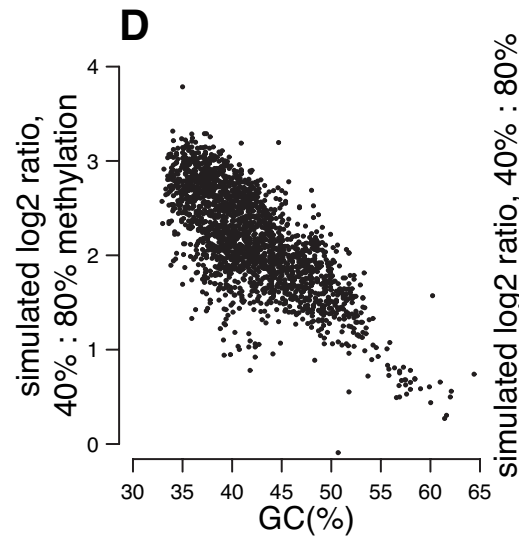

G

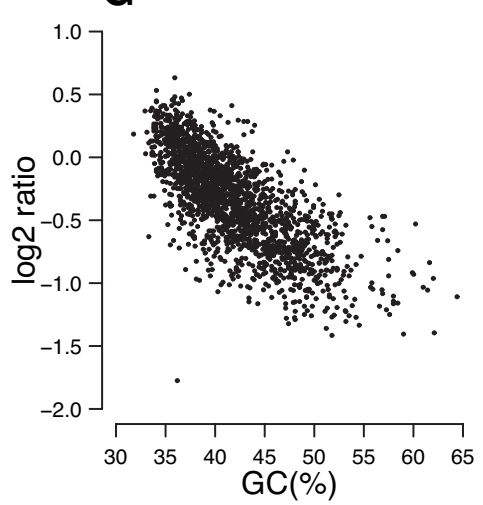

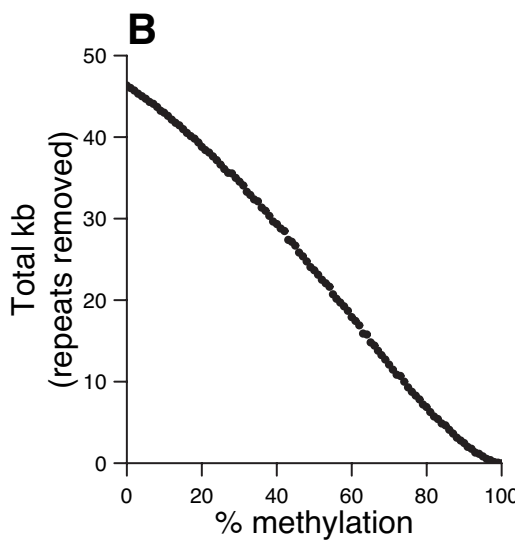

E

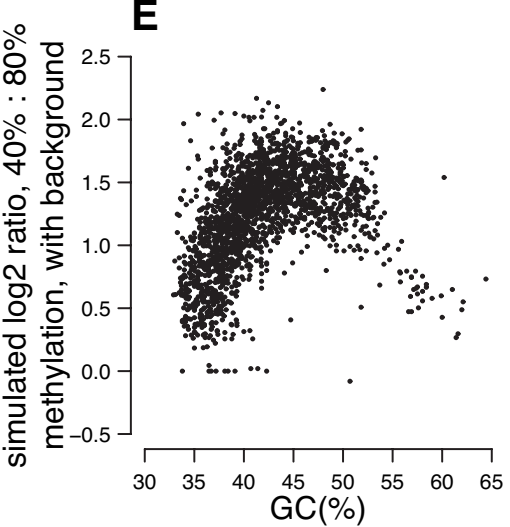

H

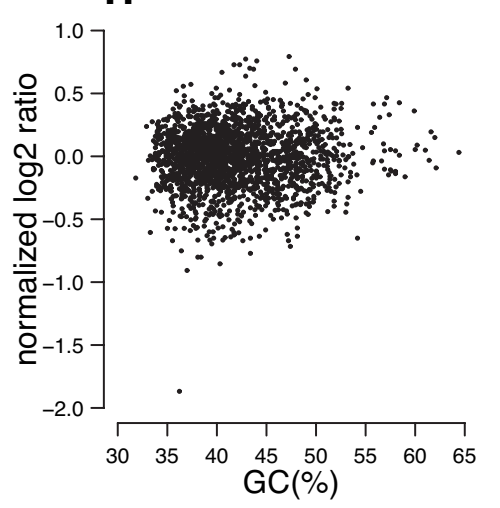

\section{Figure 2}

Fragment yield simulations. (A). The same methylation ratio ( $\mathrm{I}: 2)$ can give different measured ratios for $\mathrm{BACs}$ of different GC content. Horizontal lines represent the genomic region of a BAC in a single hybridizing sample. Sample 2 is twice as methylated as sample I. The upper two rows represent a BAC of higher GC content than the lower rows. Vertical ticks represent unmethylated (digestable) Hpall sites; check marks denote Hpall fragments passing the size filter. Hpall sites methylated in sample 2 but not sample I are marked with letters (see text). (B) Simulations based on BAC RPI I-47A4. The amount of hybridizing material decreases with increasing methylation. (C) Simulation results for all array BACs. Contour plots summarize the amount of hybridizing material for all BACs at $40 \%$ methylation (black contours) and $80 \%$ (gray). (D) Predicted ratios when samples of $40 \%$ (test) and $80 \%$ (reference) methylation are co-hybridized. (E) A more complex scenario results in an opposite relationship between GC content and ratio. In this scenario, each BAC is simulated to report cross-hybridizing material in both test and reference channels equal to $5 \%$ of the number of bases of repetitive DNA it contains. (F) Real data for a selected array (male liver:spleen) show that, as predicted, the amount of hybridizing material is higher for higher GC content BACs (liver intensities, black contours; spleen, gray). (G) Measured ratios from the same array (liver:spleen) also have a strong relationship with GC content, as predicted if samples have different overall methylation levels. (H) GC-content-normalized ratios for the same array. 
all clones on the array, but the ratios are negatively correlated with clone GC content, even though simulated methylation levels were uniform (Figure 2D). The slope of this relationship between $\log _{2}$ ratio and $\mathrm{GC} \%$ is influenced by choice of overall methylation levels simulated for the two samples, relative simulated levels of background signals on the array spots (for example, a fixed amount of non-specific signals or signals due to incompletely suppressed repetitive elements), and allowance for variation of actual methylation levels with $\mathrm{GC} \%$. In fact, some combinations of these variables can yield a generally positive (but curved) slope in the relationship between $\log _{2}$ ratio and GC\% (Figure 2E). To demonstrate this effect, we simulated adding cross-hybridizing signal proportional to each BAC's repeat content to both test and reference channels. The predicted ratio for BACs of low GC content is greatly reduced, because the cross-hybridizing material (equal for test and reference) outweighs the small amount of specific hybridizing material. BACs of higher GC content receive much more specific hybridizing material, so the cross-hybridizing material has less effect and the ratios are much closer to those predicted without cross-hybridization (compare Figures 2D and 2E).

Experimental data confirm a dependency on GC content. Note that we do not know the overall average levels of methylation for any of the samples we used, but any overall difference in methylation levels between the two samples being compared would result in a correlation between GC content and measured log ratio. In the example provided in Figure 2F, a comparison of liver and spleen tissue from the same donor, both test (liver) and reference (spleen) signal intensities for clones on the array increase with increasing clone GC content, and the observed $\log _{2}$ ratios decrease with clone GC content (Figure $2 \mathrm{G}$ ). This plot shows raw ratios, but ratios normalized by intensity, a common normalization method, show a similar relationship (not shown). Because much of the variance in $\log _{2}$ ratios across the genome is accounted for by variation in GC content, we normalized $\log _{2}$ ratios for each clone based on its GC\% using a loess fit of the relationship between $\log _{2}$ ratio and GC\% measured for each array experiment. All subsequent plots and analyses use GC-normalized values. Note that this normalization, while necessary to correct for clone-to-clone differences in measured ratios due to differences in GC content, excludes an ability to detect any true variation in methylation levels that might correlate with GC content. The GCnormalized $\log _{2}$ ratios allow us to identify potential differences in relative methylation levels for clones with similar GC content (Figure 2H).

\section{Tissue differences in methylation}

We next used the profiling method to detect methylation differences between tissues from the same individual. We evaluated lung, heart, liver, four regions of the brain (cer- ebellum, medulla oblongata, occipital lobe and pons), each from two individuals, as well as testis or ovary from single individuals. Each tissue was directly compared with spleen from the same individual to control for any interindividual variation in genomic content (for example, segmental copy number variants and/or restriction fragment length polymorphisms). We also conducted conventional array-comparative genomic hybridization (CGH) analyses on the tissue samples to verify that no significant somatic differences in copy number existed within the same individual for sequences represented by our clone array (data not shown). The female lung sample was excluded from further analyses, because it had an unusually noisy genomic copy-number profile, suggestive of random genome instability, with relative copy number correlating strongly with methylation profile ratios (data not shown).

To test the reproducibility of the method, we performed each tissue comparison in triplicate using tissues from each of two donors. The test replicates were taken from the same tissue DNA preparation and independently digested, size-selected, labeled, and hybridized to the array with similarly processed, but differentially labeled, spleen DNA. Replicate arrays for a given tissue comparison also usually employed different, independently processed replicates of the donor's spleen reference DNA (see Additional file 1). The $\log _{2}$ ratios for the replicates, both within and between donors, correlate very well for all tissue comparisons except those involving the male lung (Figure 3, Additional files 2, 3, 4, 5; summarized in Table $1)$. The poor correlation of replicate values of the three male lung-versus-spleen arrays (Pearson $R^{2}$ of 0.22 to 0.59 ) is likely because almost all of these values are close to zero, and variation is therefore predominantly experimental noise. Apart from lung, we find high Pearson correlation coefficients $\left(R^{2}\right)$ for within-donor replicates (median 0.88 , range 0.61 to $0.95, n=42$ ) (Table 1 ). Thus, profiles are robustly conserved across technical replicates of both test and reference DNA. Correlation coefficients are also high when ratio values for the same tissue from different donors are compared (median 0.66, range 0.30 to $0.86, n=54$ ) (Table 1 ). Figure 3 shows as examples the high correlation of $\log _{2}$ ratios from the cerebellum-versusspleen arrays obtained using tissues from two individuals, one male and one female (Pearson $R^{2}=0.92$ comparing replicates from the same donor, and $R^{2}=0.86$ comparing samples across donors).

Tissues show distinctly different methylation patterns along chromosome 1 (Figures 4 and 5). In each plot, we have highlighted clones that deviate from the overall median by $>4.88$ median absolute deviation (MAD) units, with MAD calculated using the data for clones in a region of $1 \mathrm{q}$ that showed the least biological variation within and across experiments (see Methods). At one 
extreme, cerebellum shows a large variation in intensity ratios from region to region when compared with spleen for both donors. Regions with the greatest dynamic range in GC-normalized ratios are distributed across the chromosome, but tend to be GC- and gene-rich regions abundant in HpaII sites (Additional file 6). These characteristics could potentially contribute to a greater dynamic range of measured ratios than possible in other regions of chromosome 1 . At the other extreme, lung and spleen are very similar, with the profiles showing only a few sites of significant difference (Figure 4).

The methylation profiles of the various lobes of the brain map are highly correlated, with many of the peaks and valleys in the profile landscapes mapping to the same chromosomal domains (Figure 5). This similarity is best appreciated in the XY-plots provided in Additional files 2, 3,4 , their correlation coefficients summarized in Table 1 , and the hierarchical cluster plots in Additional file 5 . These pronounced common differences might be the cumulative result of many genes in these regions that are regulated in a tissue-specific manner, in this case brain- or spleen-specific. The median Pearson correlation coefficient of ratios measured for the four different brain portions (each compared with spleen) from the same donor is 0.45 (range 0.27 to $0.93, \mathrm{n}=108$ ). This level of correlation approaches what we observed when comparing the same tissue taken from different individuals (see above).

In sharp contrast to the similarity of the brain tissue profiles, we observe gross differences between the methylation profiles of other tissues, perhaps reflecting regions that are differentially regulated between tissues (Figure 4, Additional file 4). The correlation coefficients are notably low when ratios measured for different organs (each relative to spleen) of the same donor are compared (median $R^{2}=0.1$, range 0 to 0.44 excluding lung and including a only single brain part (cerebellum) in these comparisons, $n=108$ ) (Table 1). Thus, methylation profiles of the same tissue in different individuals correlate more strongly than do those of different tissues from the same individual.

The heart-versus-spleen methylation profiles identify an intermediate number of clones that deviate reproducibly and significantly from the median ratio in independent comparisons (Figure 4, Additional files 2 and 7). Many of these clones were not identified as outliers in other tissue comparisons, suggesting a real difference in the methylation status between heart and spleen in these genomic regions. Below, we focus our bisulfite sequencing assays of methylation state on two of these clones with outlying values.

\section{Bisulfite confirmation of methylation differences outside of CpG islands}

We conducted bisulfite sequence analyses to confirm methylation differences between heart and spleen for sequence represented by RP11-47A4 (AL391809), which showed very high heart-versus-spleen $\log _{2}$ ratios in all replicates of both donors (Additional files 8 and 9). These very high relative heart:spleen ratios are predicted to reflect significantly less methylation of CpGs, and thus more frequent cutting with HpaII, in heart than spleen in this locus compared with other regions of chromosome 1 after accounting for
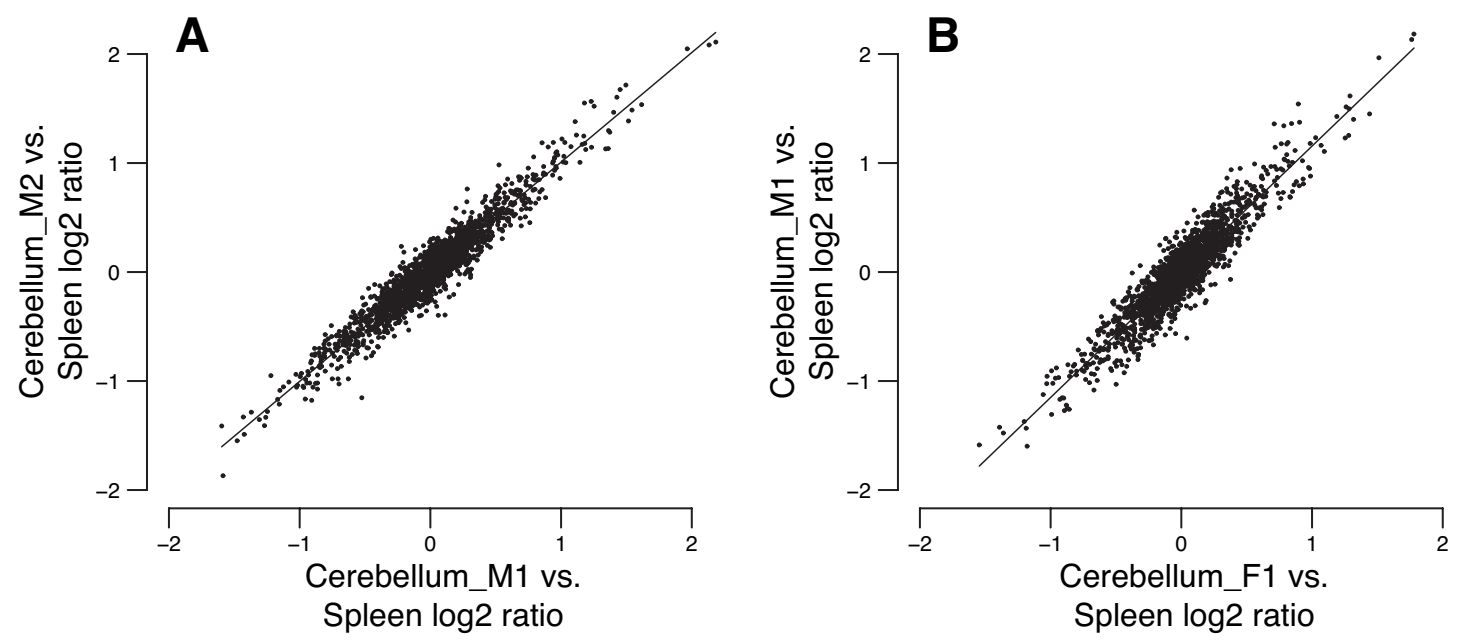

Figure 3

Intra- and inter-individual reproducibility of methylation profiling method. A very strong correlation is observed between cerebellum:spleen $\log _{2}$ ratios measured using samples from the same donor $\left(\mathrm{A}\right.$, Pearson $\left.R^{2}=0.92\right)$ and from different donors $\left(\mathrm{B}\right.$, Pearson $\left.R^{2}=0.86\right)$. See also Additional files $2,3,4$ and 5. 
Table I: Pearson correlation coefficients, $R^{2}$.

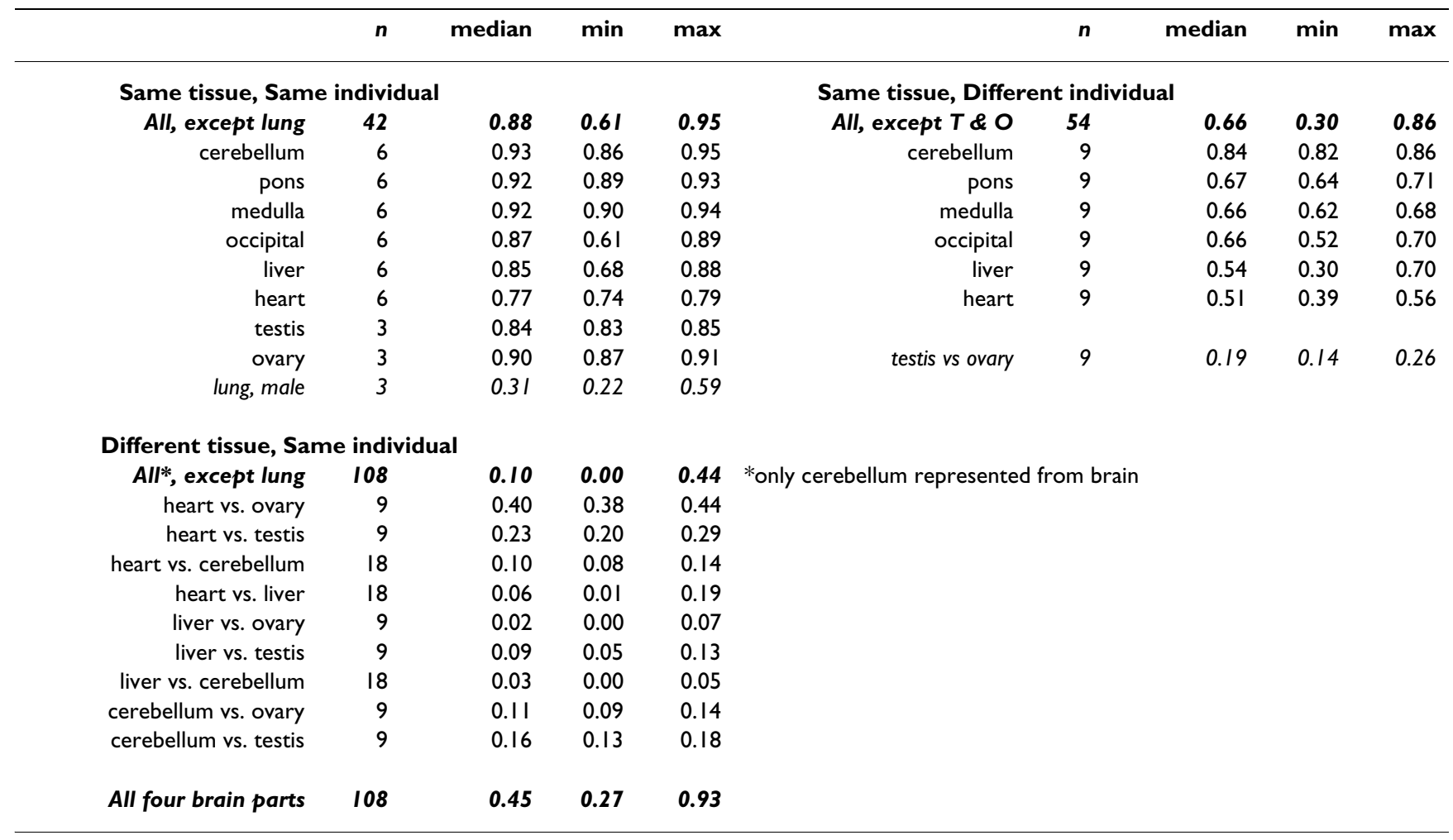

fluctuations in GC content. This clone contains part of a heart-specific gene, RYR2, making it an interesting candidate for confirmation of this prediction by bisulfite sequencing. These analyses also confirm our simulations, which predict that the method is most responsive to methylation differences outside of CPG islands.

RP11-47A4 encompasses the first exon and part of the first intron of the RYR2 cardiac receptor gene and this gene's CpG island (Figure 6, Additional file 8); it contains no other known gene. This clone gave a high ratio only in arrays for heart versus spleen ( $>9$ MAD units above the median) and cerebellum versus spleen (4 to 8 MAD units above median). RYR2 is expressed at high levels only in the heart and to a much lesser extent in some parts of the brain, albeit not cerebellum [58]; its disruption causes heart defects in humans [59-61]. We characterized the methylation state in heart and spleen of $27 \mathrm{CpGs}$ distributed across this clone's sequence outside of the $\mathrm{CpG}$ island (Figure 6, top; Additional file 9). Seven of these CpGs are in HpaII recognition sites. These HpaII sites were selected for analysis from among the 174 predicted HpaII sites in AL391809, because a change in their methylation status would have a large impact on yield in our procedure. The methylation state of these HpaII sites would determine whether or not a fragment of 500 to 2,000 bp containing $\geq 50 \%$ unique sequence would pass the size filter (when unmethylated) or be left joined to a large flanking fragment and not contribute to the hybridization signal (when methylated). We treated DNA samples with bisulfite, amplified regions of interest by PCR using primers designed to complement bisulfite-converted sequence, and sequenced cloned amplicons from heart and spleen, respectively. Our analyses of clones of bisulfite-converted DNA show that, indeed, all but one of these seven dispersed HpaII sites in AL391890 are hypomethylated in heart compared with spleen, with overall average methylation per site of $38 \%$ in heart compared with $71 \%$ in spleen (Figure 6, top; Additional file 9). Similar results were found for 20 nearby CpGs in the sequenced amplicons that are not part of HpaII sites (Figure 6, top; Additional file 9)

Although our simulations predict that the differences between heart and spleen detected by the array are dominated by methylation differences such as those outside of CpG islands, we nevertheless examined the methylation state of RYR2's CpG island, which contains regulatory elements, GC boxes essential for transcription of this gene, and spans a total of $181 \mathrm{CpGs}$. We successfully amplified two portions of the CpG island, containing a total of 53 CpGs. Overall, CpGs in the island were less methylated than CpGs distributed outside of the island in both heart and spleen. CpGs in the first 251-bp CpG island amplicon (P1) just upstream of the GC-boxes are approximately 3.5-fold less often methylated on average in heart than spleen, but the second 303-bp amplicon (P2) containing $35 \mathrm{CpGs}$, including five HpaII sites just downstream of the 


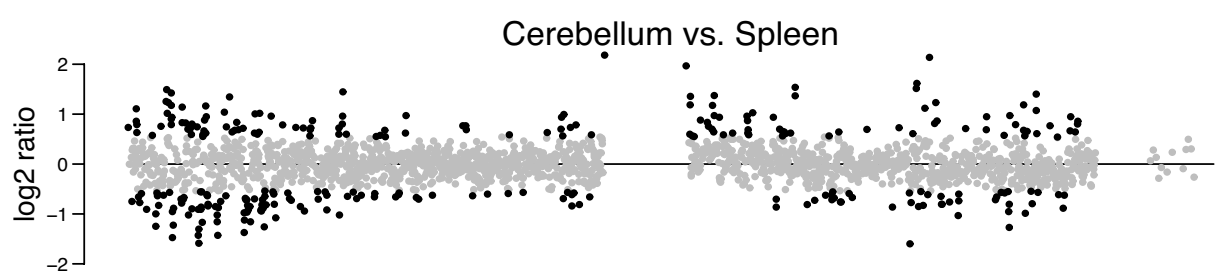

Heart vs. Spleen

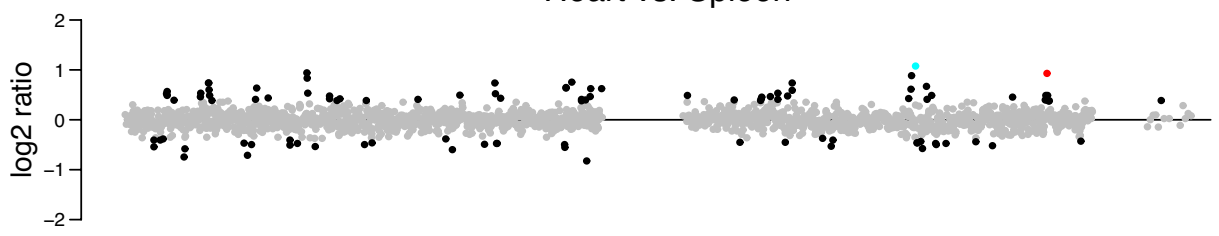

Liver vs. Spleen

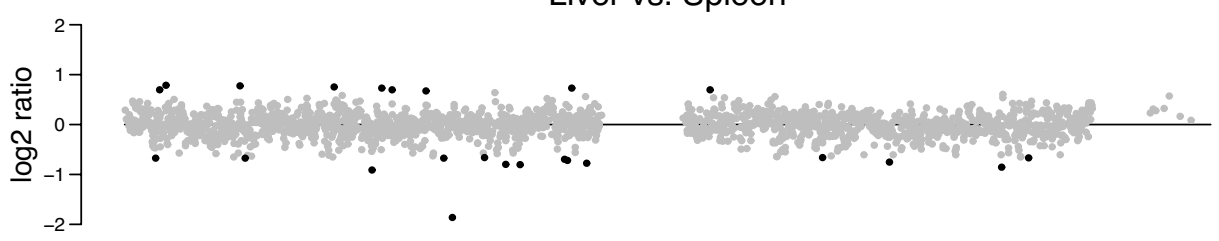

Lung vs. Spleen

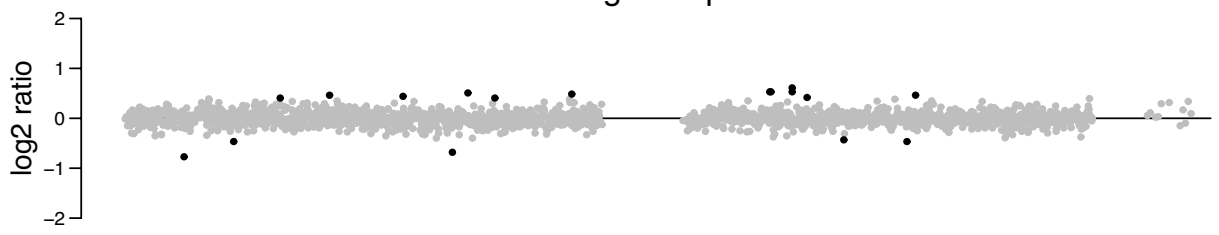

Testis vs. Spleen

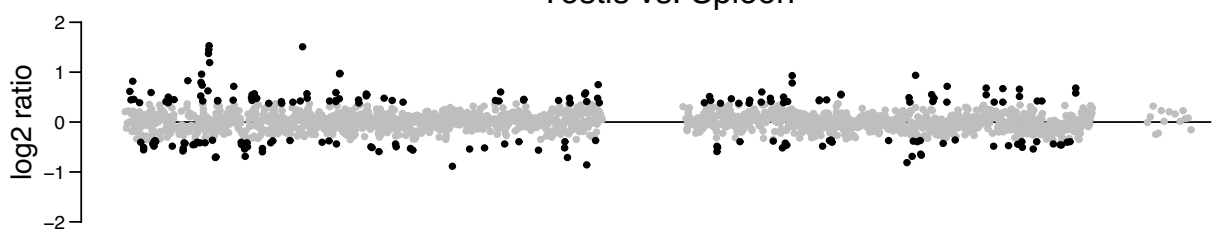

Ovary vs. Spleen

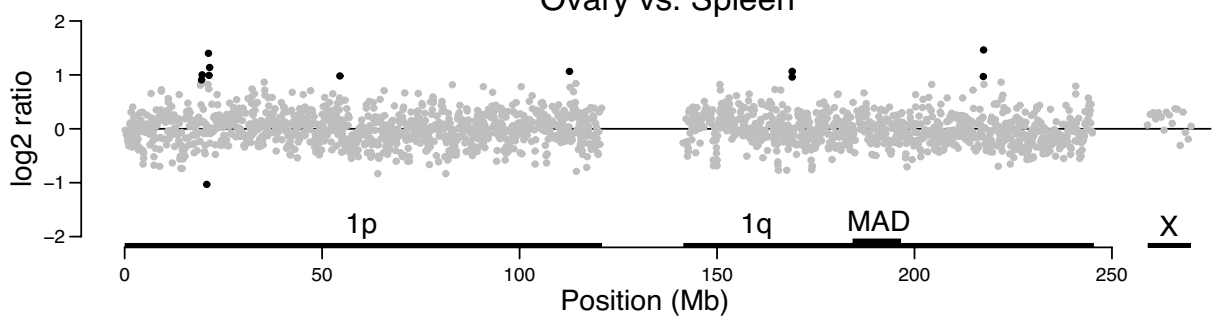

\section{Figure 4}

Methylation profiles of multiple tissues show striking differences. Cerebellum, heart, liver, lung, testis, and ovary are each profiled relative to spleen. With the exception of the ovary:spleen comparison, these representative plots were obtained using tissues from the same male donor. The $\log _{2}$ ratios for chromosome I clones are indicated at their relative genomic position, with the large gap representing the centromere and pericentromeric repeats in IpI I-Iq2I. The horizontal scale for the $\mathrm{X}$ chromosome is compressed; the 17 chromosome $\mathrm{X}$ clones on the array are actually distributed over II0 Mb. Clones highlighted in black have $\log _{2}$ ratios that deviate from the overall median, set here to 0 , in either direction by $>4.88$ median absolute deviation (MAD) units, where MAD units are calculated using the data for the clones in the region of Iq marked 'MAD' (position 184 to $197 \mathrm{Mb}$ ), which showed the least biological variation within and across experiments. High log 2 ratios are expected for regions hypomethylated relative to clones of similar GC content in the test tissue relative to spleen, whereas low log 2 ratios are expected to represent relatively hypermethylated regions. In the heart-versus-spleen profile, bacterial artificial chromosome (BAC) RPI I-47A4, containing part of the RYR2 gene, is highlighted in red and BAC RPII-90O23, containing part of $A T P 2 B 4$, is highlighted in blue. 

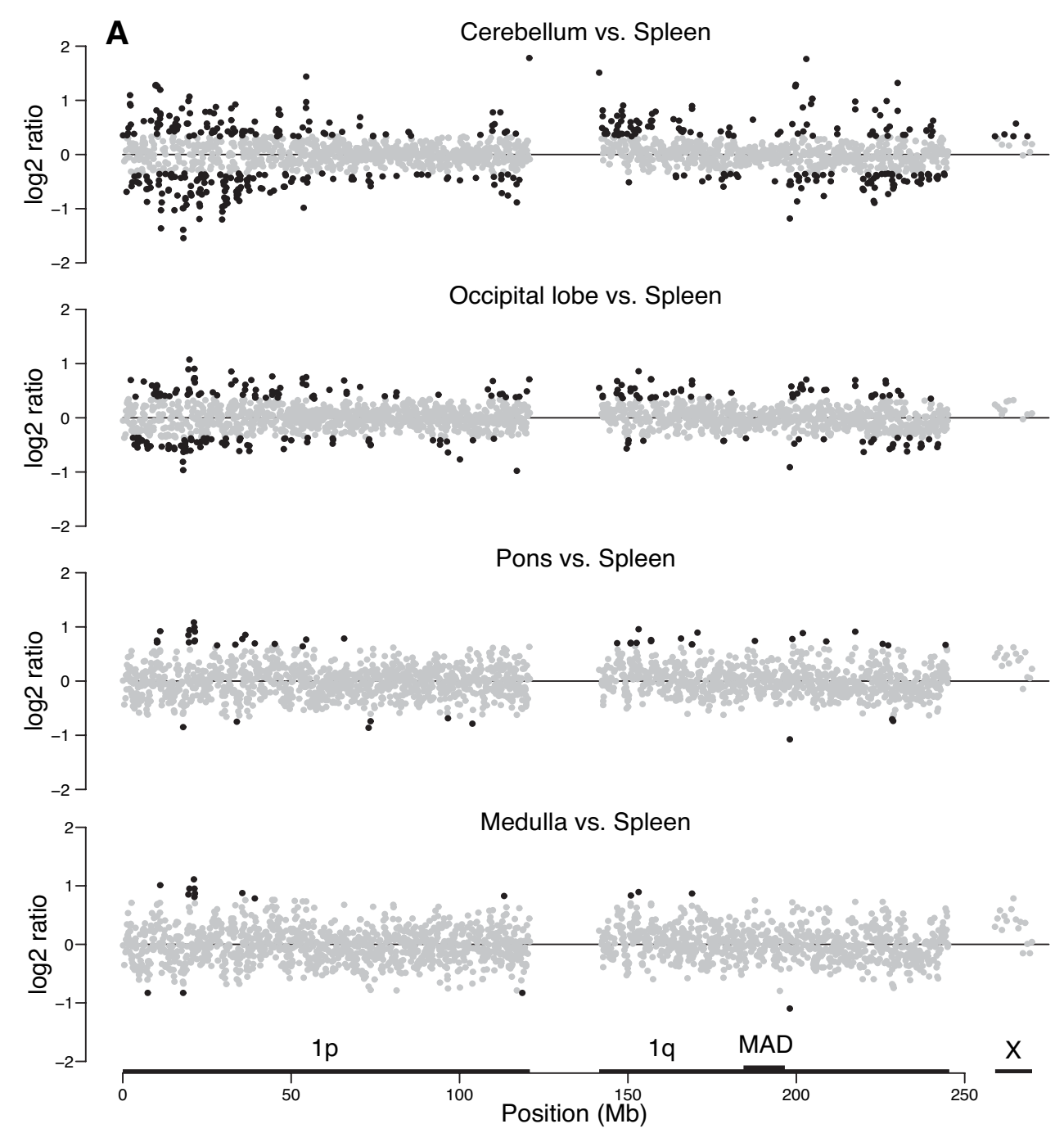

B

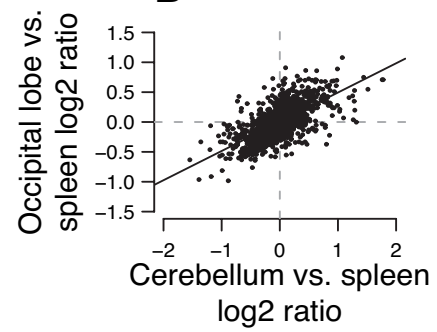

C

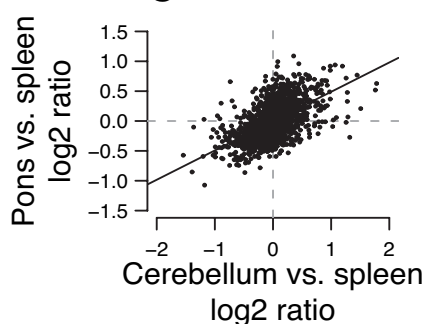

$\log 2$ ratio

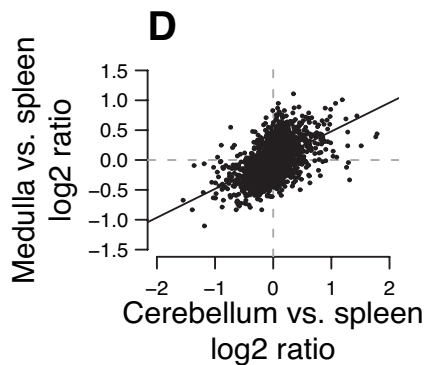

\section{Figure 5}

Methylation profiles of four lobes of the brain are similar. (A) Methylation profiles of four different brain regions from a female donor, each measured using the same individual's spleen as a reference. Clones highlighted in black have $\log _{2}$ ratios that deviate in either direction from the median (set at 0 ) by $>4.88$ median absolute deviation (MAD) units calculated from the region in Iq marked 'MAD'. See Figure 4 for additional details. (B and C) X-Y scatter plots illustrating the positive correlation between $\log _{2}$ ratios measured for different brain parts from female donor (each relative to her spleen). (B) Plot displaying a comparison of occipital lobe-versus-spleen and cerebellum-versus-spleen ratios from the female donor (FI replicates in all cases); (C)Plot showing a comparison of pons-versus-spleen and cerebellum-versus-spleen ratios; (D)Plot showing a comparison of medulla-versus-spleen and cerebellum-versus-spleen ratios. Pearson correlation coefficients $\left(R^{2}\right)$ are $0.43,0.3 \mathrm{I}$ and 0.29 for $\mathrm{B}$, $C$ and $D$, respectively, and indicate significant association at the $P<10^{-15}$ level. 

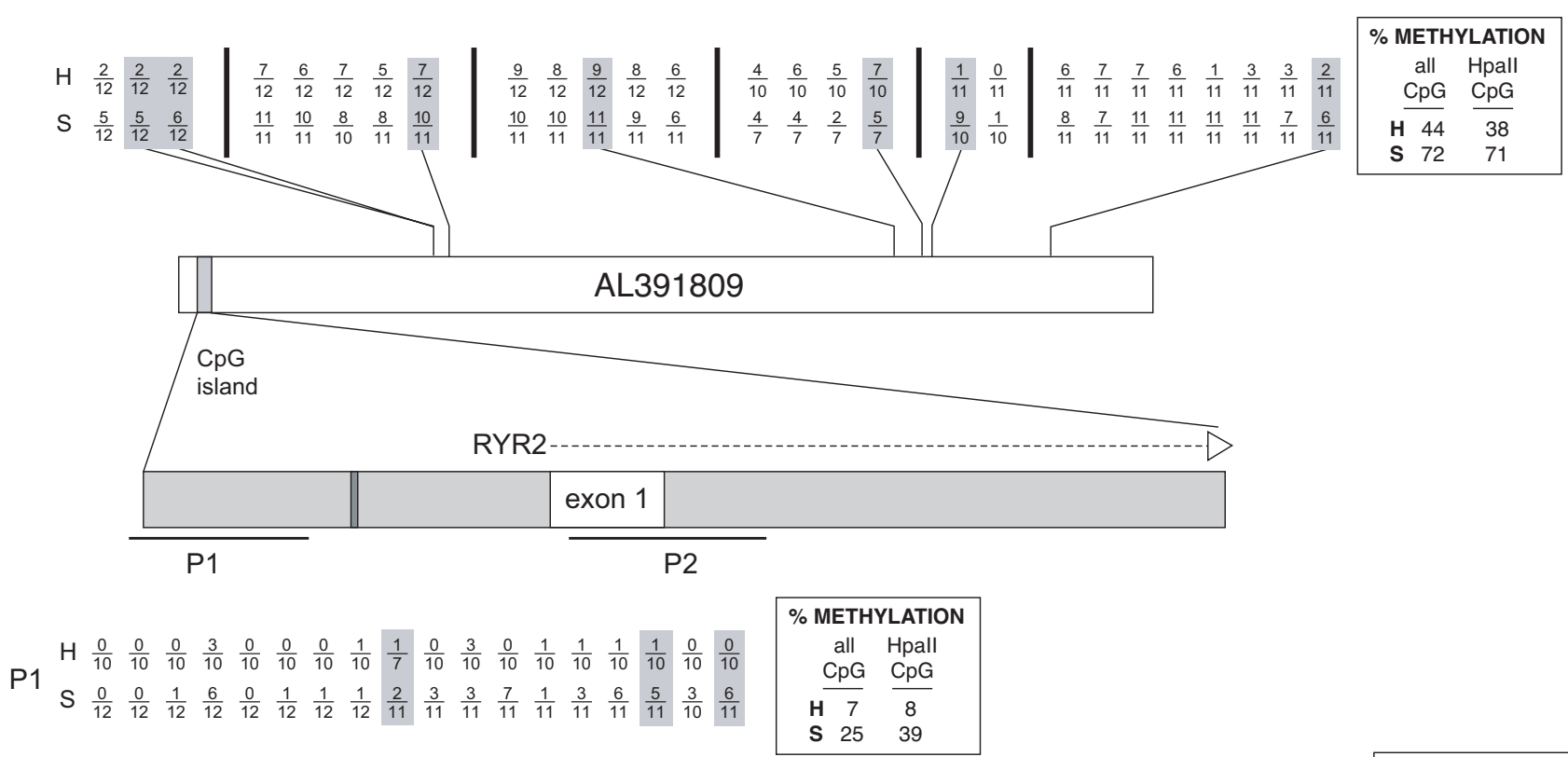

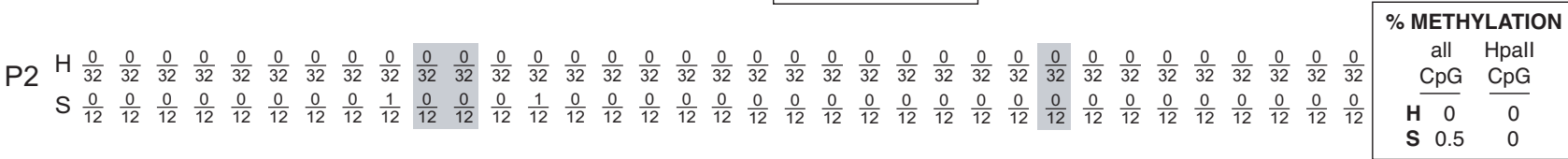

\section{Figure 6}

Summary of bisulfite sequencing analyses of male heart (H) and spleen (S) DNA in the RYR2 gene sequence represented by BAC clone RPI I-47A4 (AL39 I 809). More striking methylation differences between these tissues are observed at dispersed CpGs outside of the CpG island (top) than in the island (bottom). Each CpG analyzed by bisulfite sequencing is represented by a fraction in which the denominator is the total number of sequenced clones of PCR products amplified from bisulfite-converted DNA, and the numerator is the number of times methylation was detected at that site. Data for CpGs contained within Hpall sites (CCGG) are shaded gray. The results for distributed CpG sites not included in CpG islands are indicated along the top, with their relative positions in the AL39/809 sequence indicated. Vertical bars separate the sites on different PCR amplicons. The CPG island is highlighted by the gray vertical bar in the schematic of the AL39/809 sequence and expanded below to indicate the position of exon I of RYR2 gene (white) and the GC boxes (dark gray). Two PCR products ( $\mathrm{PI}$ and $\mathrm{P} 2$ ) containing multiple $\mathrm{CpGs}_{\mathrm{p}}$ within this $\mathrm{CpG}_{\mathrm{P}}$ island were analyzed by bisulfite sequencing, and the results are indicated at the bottom of the figure. Boxes contain overall average methylation levels for heart $(H)$ and spleen $(S)$ for CpGs sampled outside of the CpG island (top) and for CpGs sampled within the CpG island (bottom).

GC boxes, was almost entirely unmethylated in both heart and spleen (Figure 6, bottom; Additional file 9). Only 0\% and $0.5 \%$ of assayed CpGs in P2 were methylated in heart and spleen, respectively. Thus, if these combined results apply to the entire $\mathrm{CpG}$ island, methylation differences outside of the CpG island must account for most of the high signal ratio reported by this clone.

We also find no significant methylation differences between heart and spleen in the CpG island of ATP2B4, which is partially contained in RP11-90O23, another clone exhibiting a high $\log _{2}$ ratio in the heart-versus-spleen array comparisons (Additional file 7). ATP2B4 (PMCA4b) is involved in calcium homeostasis and has a role in controlling cardiac hypertrophy in response to increased load on the heart [62]. We compared the methylation status of 27 CpGs representing approximately $80 \%$ of the CpG island of ATP2B4 in heart and spleen using bisulfite sequence analysis. The $27 \mathrm{CpGs}$ were similarly unmethylated in heart and spleen (data not shown). We found average methylation levels per site of only $0.41 \%$ and $1.0 \%$ in heart and spleen, respectively. Thus, methylation differences in the region in and around ATP2B 4 must account for RP11-90O23's observed high $\log _{2}$ ratio in the heartversus-spleen comparison, as we find no methylation differences in ATP2B4's CpG island.

\section{Regions with outlying methylation ratios contain genes with outlying expression ratios}

In order to examine the possible biological significance of the methylation ratios measured by our arrays, we compared relative methylation levels with relative expression levels of corresponding genes. In all six of the tissue comparisons examined, we found that BACs with apparent 
high relative levels of methylation (that is, low array ratios) tended to contain genes that were expressed at lower relative levels than BACs with lower methylation levels. This relationship is expected if regional methylation assayed by our array, like promoter methylation, is associated with suppression of transcriptional initiation [3]. In detail, we compared our results (averaged across arrays representing the same tissue comparison) with expression data obtained by Ge et al. [58], who hybridized RNA from each of 36 normal human tissues singly to Affymetrix oligonucleotide microarrays to determine expression levels of around 20,000 human genes (see Methods). For each gene, we calculated a ratio of expression levels for six of the tissue pairs evaluated in our methylation studies, with spleen as the reference tissue in each case (Ge et al. did not generate expression data for members of the remaining tissue pairs). We compared the methylation array ratios of BAC-sized genomic regions with the expression ratios of genes whose 5 ' ends mapped within those genomic regions, recognizing that such an analysis likely ignores facets of the complex cause-andeffect relationships between the methylation levels in different parts of a gene (promoter, body of gene, and so on) with that gene's expression levels.

While the ratios reported by our methylation-sensitive arrays and expression ratios show little overall correlation (data not shown), we noticed a relationship in the extremes of the distributions: BACs reporting lower levels of methylation in the test sample than in the reference (that is, those having very high array ratios relative to others on chromosome 1 with similar GC content) tended to contain genes whose probe sets report higher expression ratios than average, and that BACs having higher implied relative methylation levels (low methylation array ratios) tended to contain genes of lower-than-average expression ratios. In order to display and test the significance of this observation, we conservatively classified the BACs into three categories reporting 'High', 'Mid' or 'Low' implied methylation levels, using the MAD-based thresholds discussed above, and examined the expression ratios of genes mapping to each of those three categories of BACs (Figure 7). Statistical tests that account for the complex many-tomany relationships in these data (see Methods) indicate that the observed increases in relative expression level with decreased relative methylation are statistically significant in most cases (Figure 7). When less conservative criteria are used for categorizing BAC methylation ratios, statistically significant relationships are seen for all six tissues comparisons (Additional file 10); the more significant results are due in part to a greater number of BACs (and therefore genes) now in the High and Low groups.

\section{Discussion}

DNA methylation is widely recognized as an epigenetic modification that plays a key role in specifying which por- tions of the genome are utilized at any given time or place in eukaryotic organisms, from plants to humans. Therefore, determining where those marks are made in the genome and how these marks are interpreted by the proteins that transcribe genes, prevent transcription of other sequences, package chromatin in the nucleus, or perform other functions on DNA, has become an important goal. We have generated chromosome-wide methylation profiles of different tissues using an approach that relies on the action of a methylation-sensitive restriction endonuclease and measurement of differences in the relative yield of size-selected fragments between two samples. Hybridization of these fragments to a tiling array of large-insert clones reports coarse regional relative differences in methylation state. Hybridization to high-resolution oligomer arrays can reveal much finer-scale fluctuations in methylation levels along the chromosome (our unpublished data).

We obtained very different methylation profiles from different organs, suggesting the existence of tissue-specific epigenetic modification patterns across chromosome 1 . These patterns are conserved across individuals, as the values measured for the same tissue obtained from different donors were strongly correlated. As one might expect given the high inter-individual similarity in methylation profiles for a given tissue, replicate analyses of the same tissue from the same donor were even more strongly correlated, demonstrating that our technique gives highly reproducible results. We find that methylation profiles of the same tissue correlate better across individuals than do profiles of different tissues from the same individual. This result corroborates findings of other studies that used alternative techniques to measure relative methylation levels $[31,33,63]$.

While most organ profiles were dissimilar, there were notable exceptions. Interestingly, the profiles for the various lobes of the brain were strongly correlated, suggesting that the shared methylation pattern of these tissues was established in a shared developmental precursor cell type and/or that the functions of these cells are sufficiently similar to be reflected in a similar pattern of epigenetic modifications. We also observe that some sites stand out as deviating from the median in multiple tissues. For example, some clones show similar relative $\log _{2}$ values in brain profiles and either testis or ovary profiles (Additional file 4). These similarities might represent spleenspecific methylation changes (spleen was used as a common reference sample) or changes common to these other organs. Other deviant sites are not shared by these different organs, such that, in fact, testis and ovary profiles are poorly correlated with each other.

Our simulations indicate that significant deviations of signal ratios from the median are influenced predominantly 

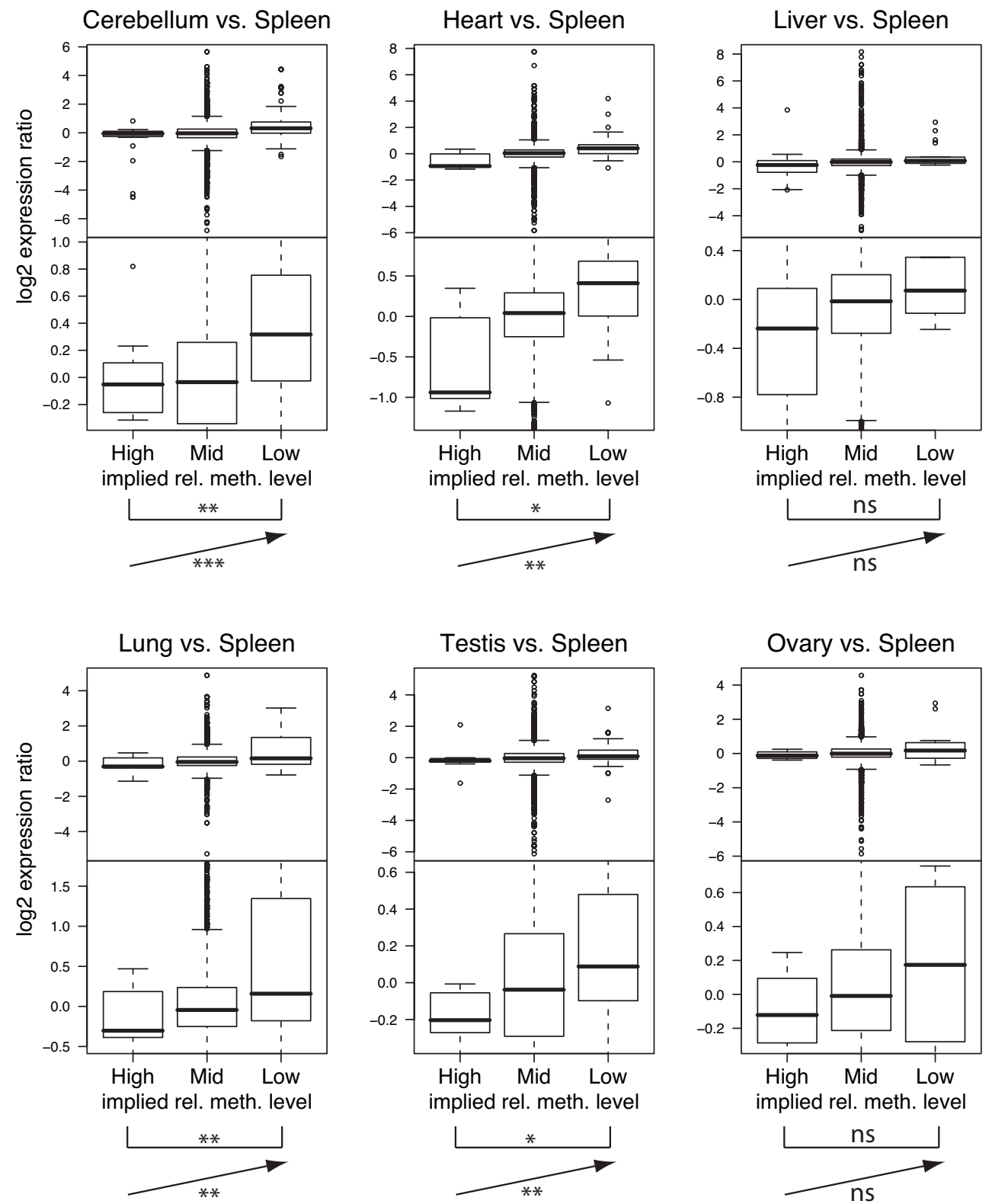

Figure 7

Expression levels are higher for genes in less methylated regions. Each pair of plots shows ratio data for a particular tissue compared with spleen, on two different $y$-axis scales to help illustrate expression differences. For each tissue comparison, we divided bacterial artificial chromosome (BACs) into three categories of implied relative methylation levels ('High', 'Mid' and 'Low') using median absolute deviation-based thresholds recalculated after averaging ratios across three technical replicates and two donors, if available. Note that low measured array ratios in a BAC-sized genomic region likely reflect more methylation in the test tissue than in spleen, relative to other regions of chromosome I with similar GC content, and are therefore labeled as 'High', and vice versa. The boxplots show the distribution of expression ratios reported by Ge et al. [58] for genes whose $5^{\prime}$ ends are in BACs of each category. On each plot, the symbol within the bracket represents the $P$ value obtained in a test of the null hypothesis that there is no difference in expression ratios of genes in BACs with 'Low' implied relative methylation levels versus BACs with 'High' relative methylation levels $(* * *, 0<P<0.001 ; * *, 0.001<P<0.01 ; *, 0.01<P<$ 0.05 ; ns, $P>0.1$, see Methods). The symbol next to the arrow represents the $P$ value obtained in a trend test of whether expression ratios are linearly related to methylation category (coding 'High', 'Mid' and 'Low' categories as - I, 0 and I, respectively). 
by methylation differences in CpG dinucleotides in HpaII sites outside of CpG islands. Thus, this method complements approaches that focus on the methylation states of CpG dinucleotides in CpG islands. Our bisulfite sequencing results support the predictions of these simulations. For example, many more of the significant methylation differences that might explain the high heart:spleen signal ratio observed for the RYR2 locus, a gene with heart-specific function, were found outside of $\mathrm{CpG}$ islands than in them. An important conclusion from these results is that CpG islands and flanking DNA can have different methylation patterns, and that tissue-specific genes can be differentially methylated in regions other than their CpG islands. This conclusion is similar to that drawn from other studies which found that both the bodies and promoters of highly expressed genes are differentially methylated compared with inactive genes $[30,31,38,50]$.

Together, our observations point to strikingly different patterns of methylation at HpaII sites outside of CpG islands in various tissues. Methylation differences in the non-CpG-island compartment of the genome are largely unappreciated and merit further study to understand their possible functional consequence(s). Of all the tissues analyzed, cerebellum showed the greatest fluctuations in relative fragment yield along the chromosome, perhaps reflecting gross variation in the density of brain-expressed genes across the chromosome. In contrast, the profiles for liver, heart and lung showed much less regional variation with respect to spleen, suggesting a more even distribution (or lack) of genes expressed specifically in these tissues and associated tissue-specific epigenetic modifications.

Our findings are consistent with recent studies that have found a comparatively low frequency of tissue-specific differentially methylated regions associated with $\mathrm{CpG}$-island promoters [33], while differences have been detected in CpG-poor promoters [26], outside CpG-dense promoters [36], and in CpG islands far from any known transcription start site [64]. Our data also reinforce the conclusions of Khulan et al. [32], who used a different approach (HELP) on mouse tissue samples to find that tissue-specific differentially methylated regions are frequent and not confined to gene promoter regions. Applying yet another methylation profiling method (RLGS), two groups found a highly significant enrichment of differentially methylated NotI sites away from CpG islands in mouse tissues $[48,65]$. Interestingly, a study by Oakes $e t$ al. found 8 -fold more hypomethylated NotI sites (among 2,600 analyzed) in testis than somatic tissues, and found relatively few brain-specific differentially methylated sites [65]. In contrast, we detect many more apparently differentially methylated regions in cerebellum and other brain parts than testes when both are compared with spleen.
These observations raise the possibility that hypomethylation of $\mathrm{CpGs}$ outside of $\mathrm{CpG}$ islands might correlate with tissue-specific transcriptional activity of genes or the great many non-canonical transcripts increasingly being recognized [66]. Indeed, when we compare our measures of relative methylation of genomic clones on the chromosome 1 array and relative expression levels of constituent genes measured for the same tissue types in other individuals by Ge et al. [58], we find that the subsets of array clones with $\log _{2}$ values above or below our MAD-defined thresholds (implying relatively low or high methylation, respectively) tend to include genes with higher or lower relative expression levels, respectively. It is worth noting that the direction of this trend is similar to that found for promoters, not gene bodies, according to recent large-scale studies that found gene bodies to be more highly methylated in highly expressed genes than in inactive genes $[31,38,50]$. The regional methylation differences we observe here might correlate with the density of other marks of active/inactive chromatin as proposed by Eckhardt et al. [33] and/or reflect (or influence) regional compartmentalization within the nucleus [67]. Alternatively, methylation of dispersed CpGs might serve to suppress spurious transcription from cryptic promoters (as proposed for Arabidopsis [54,68]), raising the interesting question as to why the relative level of protection by methylation might vary among tissues.

In conclusion, the methylation landscapes that we have generated for various tissues suggest a complex pattern of, and possible function for, methylation differences outside of CpG islands. Future studies of regional methylation, regional transcriptional activity, and large-scale organization of the nucleus will help further our understanding of these regional epigenetic differences.

\section{Materials and methods Tissue samples}

Two sets of nine tissues derived from two phenotypically normal adult individuals, one female and one male, were provided by the NCI-funded Cooperative Human Tissue Network http://chtn.nci.nih.gov[69] (CHTN-32364 and CHTN-32505, respectively).

\section{Digestion, size-fractionation, and labeling of DNA samples} We used spleen as the reference in all tissue comparisons to keep the denominator for each clone's ratio roughly constant across our experiments. Spleen DNA was used as reference for the intra-individual tissue comparisons because it was the tissue for which DNA was most abundant for both donors. Phenol- and chloroform-extracted and then isopropanol-precipitated DNA derived from test and reference tissue samples were divided into tubes $(20$ to $60 \mu \mathrm{g}$ per tube) and independently processed and analyzed. Each DNA sample was digested to completion with 
the methylation-sensitive restriction enzyme, HpaII (New England Biolabs). We monitored completion of digestion by agarose gel electrophoresis (data not shown). The digested DNA was fractionated by size on $5 \%$ to $30 \%$ sucrose gradients by using a Beckman SW-40TI swing-out rotor as previously described [53]. Fractions containing fragments smaller than $2.5 \mathrm{~kb}$ and greater than $80 \mathrm{bp}$ as judged by gel electrophoresis were selected, pooled, and precipitated with isopropanol (Figure 1). The resulting DNA was analyzed by agarose gel electrophoresis to confirm that this process had effectively selected fragments 80 to 2,500 bp in length (data not shown). One microgram each of digested, size-selected test and reference DNA was differentially labeled with Cy3-dCTP (green) or Cy5-dCTP (red) (both GE Healthcare), respectively, by random priming using the Bioprime DNA Labeling System (Invitrogen).

\section{Chromosome I tiling array hybridization}

Preparation and validation of the human chromosome 1 genomic-clone microarray was previously described [55,70]. Briefly, a total of 2,136 large-insert genomic clones were spotted in duplicate onto a glass slide. For simplicity, we will refer to this array as a 'BAC array', although the spotted clones include BACs, PACs, fosmids, and cosmids. Of these clones, 2,049 represent a tiling path covering $213 \mathrm{Mb}$, approximately $96 \%$ of the euchromatic regions of chromosome 1 . Seventeen clones represent sparsely spaced segments of the $\mathrm{X}$ chromosome, with the first at $35 \mathrm{Mb}$ and the last at $145 \mathrm{Mb}$ with median spacing of approximately $6 \mathrm{Mb}$. These clones had been included on the array as controls for sex-mismatched conventional array CGH assays for other studies. The remaining 70 clones were included on the array as they were previously thought to map to chromosomes 1 or $\mathrm{X}$, but are excluded from the results we report as their chromosomal mapping is now uncertain. Clone coordinates in the May 2004 assembly of the human genome sequence (Build 35) were provided by the Sanger Center and are available upon request. These coordinates typically imply clones longer than the spans shown on the NCBI or UCSC genome browsers due to trimming of sequences during genome assembly to eliminate overlapping sequence.

Labeled test and reference samples were mixed with $85 \mu \mathrm{g}$ of Cot-1 DNA (Invitrogen) and hybridized to arrays under conditions described elsewhere [55]. Each experiment was done in triplicate using aliquots of the DNA isolated from each tissue sample, but processed independently. The replicates were independently digested, size selected, labeled and hybridized, and paired with similarly processed spleen DNA from the same donor. Replicate arrays of a given tissue usually employed different independently processed replicates of the spleen reference DNA as well (see Additional file 1).

\section{Array data analysis}

Image acquisition was performed using an Axon $4000 \mathrm{~B}$ scanner and hybridization intensities were analyzed with the GenePixPro image analysis software (both Axon Instruments). For each spot, GenePixPro gave raw intensity values with the surrounding background subtracted for each wavelength scanned $(635 \mathrm{~nm}$ and $532 \mathrm{~nm}$ for red and green channels, respectively). A custom R script, Normalization.r, was used to $\log _{2}$-transform these values, calculate the ratio of red to green fluorescence intensities, and apply a loess normalization procedure [71] based on GC content of the BACs (see below). A script then processed the output file to identify and eliminate clones whose duplicate spots reported significantly different normalized $\log _{2}$ ratios (that is, with standard deviation (SD) $>0.28$ ) or had been flagged by GenePixPro as bad spots (that is, spots having less than $80 \%$ of pixels with intensities more than one SD above the background pixel intensity in either wavelength channel). We also eliminated results for subtelomeric clone RP5-857K21, as it appeared as an outlier on almost every array. The $\log _{2}$ ratios for the two spots for each remaining clone were averaged, and the median of all the clone averages was calculated and set to zero.

Our computer simulations of the method (see below and Results) indicated that relative fragment yield at a specified methylation level, as well as predicted intensity ratios for a pair of samples having different set methylation levels, vary with the GC content of the clones on the array. Therefore, we adjusted for GC content as follows: for each block of spots on each array, a loess curve was fitted to the relationship between the BACs' GC contents (in per cent) and their raw $\log _{2}$ ratios. The loess predicted value for each BAC's $\log _{2}$ ratio based on its GC content was subtracted from its real raw log ratio to give a normalized log ratio.

Using the GC-normalized data for each array, we then calculated the MAD for a contiguous set of 97 clones in 1q31-1q32 that contains relatively few HpaII sites and showed little clone-to-clone variation in self-to-self comparisons and little deviation from the median in any of the sample comparisons. This group of clones was used in each array experiment as an internal measure of experimental noise to help distinguish potentially biologically meaningful deviations from experimental noise, which could vary from one array to another. This region of clones, whose midpoints are between positions $184,314,284$ and $196,448,439$ bp in chromosome 1 's sequence in Build 35, is marked in each methylation profile with a bar marked 'MAD'. We used the MAD value as it is relatively robust to outliers and does not assume that values are normally distributed. The MAD value was calculated by subtracting the median $\log _{2}$ ratio for these 
selected clones from each clone's $\log _{2}$ ratio to give a deviation value; the MAD value is the median of the absolute values of these deviations. The SD of a normal distribution can be estimated to be 1.48 times the MAD value. We identified clones anywhere on chromosome 1 whose average $\log _{2}$ ratio values deviated below or above the overall median by $>4.88$ MAD units (that is, $>3.29$ times the $\mathrm{SD}$ ), which would correspond to deviations with statistical significance at the predictive value of 0.001 based on a normal distribution with two-sided $P$ value. In the self-toself comparisons, we also used all clones to calculate the MAD and identify outliers beyond the $P<0.001$ cutoff.

These outlying values, marked in each methylation profile, should include loci having true methylation differences between samples, since only $0.1 \%$ of the ratios drawn from a normal distribution (that is, two false-positive clones per array) are expected to deviate from the median by $>4.88$ MAD units. However, this threshold does not exclude all false positives due to experimental variation. The 97-clone region of 1q31-32 shows less experimental variation than the rest of the chromosome even in direct comparisons of the same tissue sample (for example, female medulla in Additional file 11). Only $0.5 \%$ of the ratios in this direct comparison deviate from the median by $>4.88 \mathrm{MAD}$ units when MAD is calculated over the entire array, whereas $2.2 \%$ do when MAD is calculated over the 97-clone region.

To control for potential genomic copy number differences between test and reference genomes, we also competitively hybridized $1 \mu \mathrm{g}$ each of sonicated test and reference total DNA, mixed with $100 \mu \mathrm{g}$ of Cot1 DNA (Invitrogen), to replicas of the same microarrays using the same conditions as described above [55]. No significant deviations in copy number were observed across chromosome 1 in any tissue sample relative to the spleen sample from the same donor in these conventional array-based comparative genomic hybridization (array CGH) assays, except the female lung sample (data not shown). Because $\log _{2}$ ratios for the methylation-profiling array correlated very strongly with the wildly varying $\log _{2}$ ratios from the conventional CGH array for this sample (in isolation, each profile appeared to have large random noise), we eliminated this sample from further analysis. Genomic content abnormalities were noted for this lung sample in another study [72].

Note that it is not possible using this comparative method alone to determine what ratio represents an equivalent methylation state in test and reference DNA, as is possible with some sequencing-based methods (for example, $[36,49])$. Thus, while a normalized $\log _{2}$ ratio of 0 (the median) might represent equivalent actual levels of methylation in a comparison of two normal tissue samples, it probably would not in a comparison of cells deficient in DNA methyltransferase activity and control cells.

\section{Methylation simulation}

In order to understand the response of our array to differently methylated genomes, we developed a PERL script, METHBATCHSIM, to simulate methylation states for a sequence of interest, such as a particular clone on the array, and the corresponding yield of fragments through our methylation-profiling procedure. First, we generated a list of HpaII restriction sites on the sequence and used RepeatMasker [56] to generate a repeat-masked sequence file. METHBATCHSIM reads in the HpaII and maskedsequence files and randomly designates which restriction sites in each clone contain methylated CpGs and which are unmethylated, for a specified overall methylation proportion. Specifically, we used the rbinom function of the $\mathrm{R}$ package [73] to randomly assign methylation status of each site, using the desired overall methylation fraction (between $0 \%$ and $100 \%$, in steps of $1 \%$ ) as the 'prob' parameter, the number of restriction sites in the BAC as the ' $n$ ' parameter, and the 'size' parameter set at 1 . The sequence is then virtually digested with HpaII based on the assigned methylation states, and we determine which of the resulting fragments are within the specified size range ( 80 to $2,500 \mathrm{bp}$ ). We simulated labeling by random priming by summing the total unmasked base pairs in all fragments meeting the size-range criterion. The final output summarizes these totals, averaged over 1,000 simulation runs. We repeated this simulation using the sequence of each clone on the array.

\section{Other bioinformatic analyses}

CpG island coordinates were taken from the UCSC Genome Browser http://genome.ucsc.edu[74] CpG Island track, where CpG islands are defined as regions of $>200$ bp with GC content $\geq 50 \%$ and a ratio $>0.6$ of observed number of CG dinucleotides to expected number on the basis of the numbers of Gs and Cs in the segment. Gene coordinates were obtained from the UCSC's RefSeq track.

\section{Expression analysis}

In order to test whether the relative methylation levels we measured relate to transcription levels, we compared our results with expression data obtained by Ge et al. [58], who hybridized RNA from each of 36 normal human tissues singly to Affymetrix oligonucleotide microarrays to determine expression levels of approximately 20,000 human genes. No RNA was available to study expression levels in samples from the same donors used for our methylation studies, but because methylation array ratios were highly correlated between the two donors we studied, it seemed reasonable to assume that methylation states would be similar in the tissues of donors used by Ge et al. and, therefore, reasonable to think that if any consistent correlation between regional methylation and expression levels exists, it might be observed even if different tissue donors are used for the methylation and expression arrays. We averaged methylation array ratios for the 
same tissue comparisons (that is, across sets of six arrays for each of cerebellum, heart and liver, and across sets of three arrays for each of lung, testis and ovary, compared with spleen in all cases). We used these cross-array averages to recalculate the MAD-based thresholds used to classify BACs as having outlying methylation array values.

Starting with Ge et al.'s raw probe-level expression-array data (GEO series GSE2361), we applied standard background adjustment, normalization and log-transformation methods using the robust multi-array average algorithm from Bioconductor's affy package [75] to obtain expression levels for each probe set (a probe set is a set of around 20 oligonucleotide probes that together represent a portion of a single gene). For each of the six tissue comparisons made (Figure 7, Additional file 10), we combined normalized expression results for the relevant tissue types to obtain $\log _{2}$ expression ratios. A table of Affymetrix probe set names, their corresponding gene symbols, and genomic coordinates of those genes in Build 35 of the human genome assembly was obtained from the SCGAP Hematopoietic Stem Cells Project [76]. We then plotted the expression ratios of probe sets corresponding to genes whose 5 ' ends mapped in BACs of each three implied categories of implied relative methylation levels: 'High', 'Mid' or 'Low'. These conservative classifications correspond to BACs with ratios on the methylation-sensitive arrays that exceed MAD-based thresholds discussed above (that is, for Figure 7, 4.88 MAD units from the median, a threshold chosen such that we would expect approximately $0.05 \%$ of BACs on the array to fall in each of the Low or High categories by chance). In each boxplot, the median is indicated by the thick line, the box spans the middle two quartiles of the distribution, the small circles are outliers, and the whiskers extend to the most extreme data point which is no more than 1.5 times the length of the box away from the box.

In order to test the statistical significance of the observed differences in relative expression ratios between genes in BACs of different methylation ratio categories, we needed to account for the many-to-many relationships that exist between BACs, genes and probe sets. A BAC can contain more than one gene, a gene's 5' end can be present in more than one overlapping $\mathrm{BAC}$, and some genes are represented on the expression array by more than one probe set (located in different parts of the gene). We therefore fitted generalized estimating equations to the data (using R's geeglm function from the geepack package [77]), treating datapoints from the same BAC as groups. We also gave each BAC-probe set datapoint a weight equal to the reciprocal of the number of times its gene symbol appeared in the table of all BAC-probe set pairs (that is, a gene represented by three probe sets and whose 5 ' end is in two overlapping BACs would receive a weight of $1 / 6$ for each of its six datapoints). For each tissue comparison, we performed two statistical tests: (a) a test of whether expression ratios differ between BACs in the 'High' and 'Low' methylation categories ( $P$ values on square brackets, Figure 7 and Additional file 10); (b) a trend test for whether expression ratios are linearly related to methylation category, when methylation levels are coded as -1 (for low array ratio and 'High' implied relative methylation level), 0 ('Mid'), or 1 (for high array ratio and 'Low' implied relative methylation level) ( $P$ values on arrows, Figure 7 and Additional file 10). In each test, we used the 'Gaussian' family to model variation in expression ratios and 'independence' as the correlation structure.

\section{PCR, cloning and sequencing of bisulfite-modified DNA}

Total genomic DNA was extracted from male heart and spleen samples, and unmethylated cytosines were converted to uracil by bisulfite treatment according to published protocols [78]. Briefly, each DNA sample was first digested with Bam $\mathrm{H} 1$, the enzyme was removed by phenol and chloroform extraction, and the DNA was subsequently ethanol precipitated and resuspended in $\mathrm{H}_{2} \mathrm{O}$. $\mathrm{NaOH}$ was added to each DNA sample to a final concentration of $0.3 \mathrm{M}$, and the DNA was denatured for $2 \mathrm{~min}$ at $97^{\circ} \mathrm{C}$ followed by incubation for $30 \mathrm{~min}$ at $39^{\circ} \mathrm{C}$. A solution of sodium bisulfite and hydroquinone was immediately added to $3.3 \mathrm{M}$ and $0.67 \mathrm{mM}$ final concentrations, respectively, and samples were incubated for $16 \mathrm{~h}$ at $55^{\circ} \mathrm{C}$ with $95^{\circ} \mathrm{C}$ spikes for 5 min every $3 \mathrm{~h}$. Samples were desalted using QIAquick PCR Purification columns (Qiagen) according to the manufacturer's instructions. $\mathrm{NaOH}$ was added to each converted sample to a final concentration of $0.3 \mathrm{M}$, and the samples were incubated at $37^{\circ} \mathrm{C}$ for $15 \mathrm{~min}$ to remove excess sodium bisulfite. DNA samples were then ethanol precipitated and resuspended in $100 \mu \mathrm{H}_{2} \mathrm{O}$.

Primers for selected regions were designed using the BiSearch Web Server [79] and are provided upon request. Each amplification was done with touchdown PCR using $2 \mu \mathrm{l}$ Ex-Taq enzyme (Takara Mirus Bio) and $0.5 \mu \mathrm{l}$ of 50 $\mu \mathrm{M}$ primers in a total volume of $25 \mu \mathrm{l}$ with the following conditions: $95^{\circ} \mathrm{C}$ for $2 \mathrm{~min}, 30$ cycles of $95^{\circ} \mathrm{C}$ for $30 \mathrm{~s}$, $66^{\circ} \mathrm{C}$ for 30 s (stepping down $2^{\circ} \mathrm{C}$ every 2 cycles until $56^{\circ} \mathrm{C}$ ), and $72^{\circ} \mathrm{C}$ for $1 \mathrm{~min}$, followed by $72^{\circ} \mathrm{C}$ for $10 \mathrm{~min}$.

Amplified products from converted DNA were gel-purified with QIAquick Gel Extraction columns (Qiagen) according to the manufacturer's instructions and subsequently cloned into the pCR2.1 plasmid vector using the TOPO TA Cloning Kit (Invitrogen). Clones with inserts were identified by PCR amplification using M13 reverse and T7 forward primers using TOPO cloning instructions. Amplified M13-T7 products were purified with Sephacryl S-300 (Amersham BioSciences). Seven to thirty-two clones were then sequenced successfully from each PCR product using the same primers on an ABI 3730 (Applied Biosystems). Sequences were aligned and analyzed using PhredPhrap and Consed [80-82]. 
All array data relevant to this manuscript have been submitted to the Gene Expression Omnibus (GEO) under accession number GSE12925.

\section{Abbreviations}

ATP2B4: Ca-transporting plasma membrane ATPase isoform 4; BAC: bacterial artificial chromosome; CGH: comparative genomic hybridization; MAD: median absolute deviation; PAC: P1-derived artificial chromosome; RYR2, ryanodine cardiac receptor 2; SD: standard deviation.

\section{Competing interests}

The authors declare that they have no competing interests.

\section{Authors' contributions}

$\mathrm{CDB}, \mathrm{ER}$, and RKT carried out the methylation profiling experiments. CDB, ER, JMY, UM and BJT analyzed the array data. ER carried out the bisulfite sequencing, and ER and JMY analyzed the data. CFL was responsible for BAC array design and fabrication. EEE contributed tissue samples. LH participated in the statistical analysis. JMY performed simulation studies. JPD, SH, EEE, and BJT participated in the overall design and coordination of the study. CDB, ER, JMY, and BJT wrote the manuscript. All authors read and approved the final manuscript.

\section{Additional material}

\section{Additional File 1}

Table S1. Replicates of test and spleen reference paired for each array. Click here for file

[http://www.biomedcentral.com/content/supplementary/17568935-2-7-S1.xls]

\section{Additional File 2}

Figure S1. Scatter plots are displayed above the diagonal to illustrate pairwise comparisons between results of different methylation profiling microarray experiments. The corresponding Pearson $\mathrm{R}^{2}$ correlation coefficients are shown below the diagonal. This figure includes three replicate arrays each for various tissue samples from each of two donors. Female lung is excluded due to observed genomic copy number deviations. Only cerebellum is included in this set as a representative brain part due to space considerations. The reference tissue in each array is spleen, from the same donor as the test tissue. The red dashed line in each plot indicates the linear regression fit. Cells along the diagonal provide tissue and replicate information. Abbreviation: Cere, Cerebellum. Replicates are indicated by the suffix following the underscore: $M 1, M 2$, and $M 3$ are replicates from the male donor; F1, F2, F3 are replicates from the female donor. Most replicate arrays for a given tissue employed different replicates of the spleen reference (see Additional file 1).

Click here for file

[http://www.biomedcentral.com/content/supplementary/17568935-2-7-S2.jpeg]

\section{Additional File 3}

Figure S2. Scatter plots are displayed above the diagonal to illustrate pairwise comparisons between results of different methylation profiling microarray experiments performed using various brain tissue samples. The corresponding Pearson $\mathrm{R}^{2}$ correlation coefficients are shown below the diagonal. This figure includes three replicate arrays for each tissue sample from each of two donors. Abbreviations: Cere, Cerebellum; Occi, Occipital Lobe; Med, Medulla. See Additional file 2 legend for additional details. Plots comparing F1 brain samples are also shown in Figures 5B, 5C, and 5D.

Click here for file

[http://www.biomedcentral.com/content/supplementary/17568935-2-7-S3.jpeg]

\section{Additional File 4}

Figure S3. Scatter plots are displayed above the diagonal to illustrate pairwise comparisons between results of different methylation profiling microarray experiments performed using various tissues from two donors. Here one representative replicate of each tissue from each donor is included in the pairwise comparison dataset. The corresponding Pearson $\mathrm{R}^{2}$ correlation coefficients are shown below the diagonal. See Additional file 2 legend for additional details.

Click here for file

[http://www.biomedcentral.com/content/supplementary/17568935-2-7-S4.jpeg]

\section{Additional File 5}

Figure S4. Hierarchical clustering of methylation profiles from 45 fractionated DNA samples from 15 tissue samples. GC-normalized ratios were clustered using Manhattan distances and the Ward method of hierarchical clustering using $R$ 's hclust function. The dendrogram demonstrates that there is tight correlation within each set of triplicate samples and between samples from the same tissue in different individuals. Abbreviations: Med, Medulla; Cere, Cerebellum; Occi, Occipital Lobe. Click here for file [http://www.biomedcentral.com/content/supplementary/17568935-2-7-S5.pdf]

\section{Additional File 6}

Figure S5. Chromosome 1 G-banding, GC\%, gene density, and HpaII site distribution. The G-banding pattern of chromosome 1 is aligned to plots of (1) of the percentage of $G$ or $C$ nucleotides in each array clone as a function of the clone's position along the chromosome, (2) of the number of start sites of RefSeq genes in non-overlapping windows of 1 Mbp along the chromosome (obtained from the UCSC Genome Browser RefSeq track), and (3) the number of possible HpaII fragments in the 80 to 2,500 bp size range in each array clone as a function of the clone's position along the chromosome. The thick black bar indicates the region of 97 clones used to calculate median absolute deviation values in all experiments.

Click here for file

[http://www.biomedcentral.com/content/supplementary/17568935-2-7-S6.pdf] 


\section{Additional File 7}

Figure S6. Reproducibility of methylation profiles across three experiments in which heart DNA is compared with spleen using tissue from the male donor. DNA was extracted from a single heart and single spleen tissue sample, and each DNA preparation was then divided into aliquots that were independently processed through $\mathrm{HpaII}$ digestion, size selection, labeling, and array hybridization. Replicates are identified as $\mathrm{M} 1, \mathrm{M} 2$, and $\mathrm{M} 3$ for heart, and $\mathrm{M} 1, \mathrm{M} 6$, and $M 7$ for spleen, where $M$ denotes the male donor. (A) Profiles of $\log _{2}$ ratios as function of genomic position. Clones highlighted in black, red, or blue have $\log _{2}$ ratios that deviate from the median by $>4.88$ median absolute deviation, which was calculated over the region indicated with a wide bar. The clone whose value is indicated in red is RP11-47A4, which contains part of the RYR2 gene; the value in blue is for clone RP11-90O23, which contains ATP2B4. Sequences encompassed by these clones are the subject of our bisulfite sequencing analyses. (B) Correlations of $\log _{2}$ ratios measured in the three pairs of experiments. Scatterplots are shown above the diagonal; values below the diagonal are the corresponding Pearson correlation coefficients $\left(\mathrm{R}^{2}\right)$.

Click here for file

[http://www.biomedcentral.com/content/supplementary/17568935-2-7-S7.pdf]

\section{Additional File 8}

Figure S7. Enlarged view of clones on the array around the region containing the ryanodine cardiac receptor 2 gene (RYR2). Relative positions of bacterial artificial chromosomes (BACs) covering the region flanking RP11-47A4

(AL391809) and their corresponding mean $\log _{2}$ ratios (dotted lines are at \pm 1 standard error of the mean) in six heart-versus-spleen methylation profiles (three using DNA prepared from tissue from the female donor, and three utilizing DNA from tissue from the male donor). RP11-47A4 has the highest mean $\log _{2}$ ratio value of the five BACs shown here. The relative positions of RYR2 exons are indicated by black dots (size not to scale) and the RYR2 CDG island, which coincides with RYR2's first exon is indicated. RYR2 extends approximately $340 \mathrm{~kb}$ beyond the region shown.

Click here for file

[http://www.biomedcentral.com/content/supplementary/17568935-2-7-S8.pdf]

\section{Additional File 9}

Figure S8. Methylation status of CpGs in RP11-47A4 (AL391809), which contains the RYR2 gene, as determined by sequencing of PCR products amplified after bisulfite conversion of unmethylated cytosines to uracil. These data are summarized in Figure 6. Here, the raw data are provided for each sequenced amplicon from heart or spleen. Filled squares represent methylated CpG dinucleotides, open squares unmethylated CpGs, and gray background shading denotes $C p G$ s that are part of $\mathrm{HpaII}$ restriction sites.

Click here for file

[http://www.biomedcentral.com/content/supplementary/17568935-2-7-S9.pdf]

\section{Additional File 10}

Figure S9. Expression ratios tend to be higher for genes whose 5' ends fall in regions of lower methylation (that is, in bacterial artificial chromosomes (BACs) with higher methylation array ratios) than those in regions of higher methylation. Data are presented as in Figure 7, but here BACs are classified in the 'High' and 'Low' categories using a less conservative methylation array ratio threshold. This less conservative threshold is defined as $2.91 *$ median absolute deviation (MAD) units below and above the median value. At the $2.91{ }^{*}$ MAD threshold, we expect approximately $2.5 \%$ of BACs on the array to fall into each of the 'High' and 'Low' categories purely by chance (that is, an overall false-positive rate of $5 \%$ of array BACs). Figure 7 conservatively uses $4.88 * M A D$, such that the false-positive rate is expected to be only around $0.1 \%$ of the BACs on the array. The increased number of BACs in the 'High' and 'Low' categories using this less conservative approach likely gives the statistical tests greater power, explaining the greater significance of most $\mathrm{P}$ values shown here as compared with Figure 7.

Click here for file

[http://www.biomedcentral.com/content/supplementary/17568935-2-7-S10.pdf]

\section{Additional File 11}

Figure S10. Methylation profile in which two independently processed aliquots of DNA isolated from medulla tissue from the same female donor are directly compared. The $\log _{2}$ ratios for chromosome 1 clones are indicated at their relative genomic position, with the large gap representing the centromere and pericentromeric repeats in 1p11-1q21. The horizontal scale for the X chromosome is compressed; the 17 chromosome $X$ clones on the array are distributed over $110 \mathrm{Mb}$. Clones highlighted in black or red have $\log _{2}$ ratios that deviate from the overall median, set here to 0 , in either direction by $>4.88$ median absolute deviation $(M A D)$ units, where $M A D$ units are calculated using the data for the clones in the region of 19 marked 'MAD', which showed the least biological variation within and across experiments. Clones highlighted in red deviate from the overall median by $\pm 4.88 \mathrm{MAD}$, calculated using all values.

Click here for file

[http://www.biomedcentral.com/content/supplementary/17568935-2-7-S11.pdf]

\section{Acknowledgements}

We thank Sean McGrath and Arkadiusz Piotrowski for technical assistance with tissue and cell samples, Jeff Delrow and Cassie Neal in the FHCRC Genomics facility for technical assistance, and Peter Ellis for printing the $B A C$ arrays. This work was supported in part by a graduate fellowship from the Department of Education, Universities and Research of the Basque Government (to $C D B$ ), a Ford Foundation Predoctoral Diversity Fellowship (to ER), an NIH training grant fellowship T32-CA09657 (to RKT), the Wellcome Trust (to CFL), the Howard Hughes Medical Institute (to EEE and SH), NIH ROI HD0435679 (to EEE), the Swedish Cancer Society, Swedish Children's Cancer Foundation, and the US Army Medical Research and Materiel Command award No. W8IXWH-04-I-0269 (to JPD), and the Fred Hutchinson Cancer Research Center. This manuscript is based on work completed while ER was employed at the University of Washington. The opinions expressed here are the author's own and do not reflect the views of the Department of Health and Human Services. 


\section{References}

I. Li E, Bestor TH, Jaenisch R: Targeted mutation of the DNA methyltransferase gene results in embryonic lethality. Cell 1992, 69:915-926.

2. Goll MG, Bestor TH: Eukaryotic cytosine methyltransferases. Annu Rev Biochem 2005, 74:48I-5I4.

3. Bird A: DNA methylation patterns and epigenetic memory. Genes Dev 2002, I 6:6-2I.

4. Pfeifer GP, Tanguay RL, Steigerwald SD, Riggs AD: In vivo footprint and methylation analysis by PCR-aided genomic sequencing: comparison of active and inactive $X$ chromosomal DNA at the CpG island and promoter of human PGK-I. Genes Dev 1990, 4:1277-1287.

5. Li E, Beard C, Jaenisch R: Role for DNA methylation in genomic imprinting. Nature 1993, 366:362-365.

6. Yoder JA, Walsh CP, Bestor TH: Cytosine methylation and the ecology of intragenomic parasites. Trends Genet 1997, I 3:335-340.

7. Martienssen RA, Colot V: DNA methylation and epigenetic inheritance in plants and filamentous fungi. Science $200 \mathrm{I}$, 293:1070-1074.

8. Fraga MF, Ballestar E, Paz MF, Ropero S, Setien F, Ballestar ML, HeineSuner D, Cigudosa JC, Urioste M, Benitez J, Boix-Chornet M, Sanchez-Aguilera A, Ling C, Carlsson E, Poulsen P, Vaag A, Stephan Z, Spector TD, Wu YZ, Plass C, Esteller M: Epigenetic differences arise during the lifetime of monozygotic twins. Proc Natl Acad Sci USA 2005, 102: 10604-10609.

9. Amir RE, Veyver IB Van den, Wan M, Tran CQ, Francke U, Zoghbi HY: Rett syndrome is caused by mutations in $X$-linked MECP2, encoding methyl-CpG-binding protein 2. Nat Genet 1999, 23:185-188.

10. Brannan $\mathrm{Cl}$, Bartolomei $\mathrm{MS}$ : Mechanisms of genomic imprinting. Curr Opin Genet Dev 1999, 9:164-170.

II. Hansen RS, Wijmenga C, Luo P, Stanek AM, Canfield TK, Weemaes CM, Gartler SM: The DNMT3B DNA methyltransferase gene is mutated in the ICF immunodeficiency syndrome. Proc Natl Acad Sci USA 1999, 96:144I2-144I7.

12. Okano M, Bell DW, Haber DA, Li E: DNA methyltransferases Dnmt3a and Dnmt3b are essential for de novo methylation and mammalian development. Cell 1999, 99:247-257.

13. Nicholls RD, Knepper JL: Genome organization, function, and imprinting in Prader-Willi and Angelman syndromes. Annu Rev Genomics Hum Genet 2001, 2:153-175.

14. Sparago A, Cerrato F, Vernucci M, Ferrero GB, Silengo MC, Riccio A: Microdeletions in the human HI9 DMR result in loss of IGF2 imprinting and Beckwith-Wiedemann syndrome. Nat Genet 2004, 36:958-960.

15. Paz MF, Wei S, Cigudosa JC, Rodriguez-Perales S, Peinado MA, Huang $\mathrm{TH}$, Esteller M: Genetic unmasking of epigenetically silenced tumor suppressor genes in colon cancer cells deficient in DNA methyltransferases. Hum Mol Genet 2003, I 2:2209-22 19.

16. Rideout WM 3rd, Eversole-Cire P, Spruck CH 3rd, Hustad CM, Coetzee GA, Gonzales FA, Jones PA: Progressive increases in the methylation status and heterochromatinization of the myoD CpG island during oncogenic transformation. Mol Cell Biol 1994, 14:6|43-6152.

17. Laird PW, Jaenisch R: The role of DNA methylation in cancer genetics and epigenetics. Annu Rev Genet 1996, 30:44 I-464.

18. Esteller M: Cancer epigenomics: DNA methylomes and histone-modification maps. Nat Rev Genet 2007, 8:286-298.

19. Larsen F, Gundersen G, Lopez R, Prydz H: CpG islands as gene markers in the human genome. Genomics 1992, I 3:1095-I 107.

20. Wang Y, Leung FC: An evaluation of new criteria for CpG islands in the human genome as gene markers. Bioinformatics 2004, 20: II70-II77.

21. loshikhes IP, Zhang MQ: Large-scale human promoter mapping using CpG islands. Nat Genet 2000, 26:6I-63.

22. Prendergast GC, Ziff EB: Methylation-sensitive sequence-specific DNA binding by the c-Myc basic region. Science 1991, 25 I: I86-189.

23. Ohlsson R, Renkawitz R, Lobanenkov V: CTCF is a uniquely versatile transcription regulator linked to epigenetics and disease. Trends Genet 200I, I 7:520-527.

24. Chen C, Yang MC, Yang TP: Evidence that silencing of the HPRT promoter by DNA methylation is mediated by critical CpG sites. J Biol Chem 200I, 276:320-328.
25. Jaenisch R, Bird A: Epigenetic regulation of gene expression: how the genome integrates intrinsic and environmental signals. Nat Genet 2003, 33(Suppl):245-254.

26. Weber M, Hellmann I, Stadler MB, Ramos L, Pääbo S, Rebhan M, Schübeler D: Distribution, silencing potential and evolutionary impact of promoter DNA methylation in the human genome. Nat Genet 2007, 39:457-466.

27. Bird AP, Taggart MH, Nicholls RD, Higgs DR: Non-methylated CpG-rich islands at the human alpha-globin locus: implications for evolution of the alpha-globin pseudogene. EMBO J 1987, 6:999-1004.

28. Jones PA, Wolkowicz MJ, Rideout WM 3rd, Gonzales FA, Marziasz CM, Coetzee GA, Tapscott SJ: De novo methylation of the MyoD I CpG island during the establishment of immortal cell lines. Proc Natl Acad Sci USA 1990, 87:61 I7-6I 21 .

29. McKeon C, Ohkubo H, Pastan I, de Crombrugghe B: Unusual methylation pattern of the alpha 2 (I) collagen gene. Cell I982, 29:203-210.

30. Hellman A, Chess A: Gene body-specific methylation on the active $X$ chromosome. Science 2007, 3 | 5: | | 4|-||43.

31. Ball MP, Li JB, Gao Y, Lee JH, LeProust EM, Park IH, Xie B, Daley GQ, Church GM: Targeted and genome-scale strategies reveal gene-body methylation signatures in human cells. Nat Biotechnol 2009, 27:36I-368.

32. Khulan B, Thompson RF, Ye K, Fazzari MJ, Suzuki M, Stasiek E, Figueroa ME, Glass JL, Chen Q, Montagna C, Hatchwell E, Selzer RR, Richmond TA, Green RD, Melnick A, Greally JM: Comparative isoschizomer profiling of cytosine methylation: the HELP assay. Genome Res 2006, I 6:1046-1055.

33. Eckhardt F, Lewin J, Cortese R, Rakyan VK, Attwood J, Burger M, Burton J, Cox TV, Davies R, Down TA, Haefliger C, Horton R, Howe K, Jackson DK, Kunde J, Koenig C, Liddle J, Niblett D, Otto T, Pettett R, Seemann S, Thompson C, West T, Rogers J, Olek A, Berlin K, Beck S: DNA methylation profiling of human chromosomes 6, 20 and 22. Nat Genet 2006, 38: | 378-I 385.

34. Suzuki MM, Kerr AR, De Sousa D, Bird A: CpG methylation is targeted to transcription units in an invertebrate genome. Genome Res 2007, I 7:625-63I.

35. Weber M, Schübeler D: Genomic patterns of DNA methylation: targets and function of an epigenetic mark. Curr Opin Cell Biol 2007, I 9:273-280.

36. Meissner A, Mikkelsen TS, Gu H, Wernig M, Hanna J, Sivachenko A Zhang X, Bernstein BE, Nusbaum C, Jaffe DB, Gnirke A, Jaenisch R, Lander ES: Genome-scale DNA methylation maps of pluripotent and differentiated cells. Nature 2008, 454:766-770.

37. Lister R, O'Malley RC, Tonti-Filippini J, Gregory BD, Berry CC, Millar $\mathrm{AH}$, Ecker JR: Highly integrated single-base resolution maps of the epigenome in Arabidopsis. Cell 2008, I33:523-536.

38. Deng J, Shoemaker R, Xie B, Gore A, LeProust EM, AntosiewiczBourget J, Egli D, Maherali N, Park IH, Yu J, Daley GQ, Eggan K, Hochedlinger K, Thomson J, Wang W, Gao Y, Zhang K: Targeted bisulfite sequencing reveals changes in DNA methylation associated with nuclear reprogramming. Nat Biotechnol 2009, 27:353-360

39. Kawai J, Hirotsune S, Hirose K, Fushiki S, Watanabe S, Hayashizaki Y: Methylation profiles of genomic DNA of mouse developmental brain detected by restriction landmark genomic scanning (RLGS) method. Nucleic Acids Res 1993, 2 I:5604-5608.

40. Ushijima T, Morimura K, Hosoya Y, Okonogi H, Tatematsu M, Sugimura T, Nagao M: Establishment of methylation-sensitive-representational difference analysis and isolation of hypo- and hypermethylated genomic fragments in mouse liver tumors. Proc Natl Acad Sci USA 1997, 94:2284-2289.

4I. Gonzalgo ML, Liang G, Spruck CH 3rd, Zingg JM, Rideout WM 3rd, Jones PA: Identification and characterization of differentially methylated regions of genomic DNA by methylation-sensitive arbitrarily primed PCR. Cancer Res 1997, 57:594-599.

42. Toyota M, Ho C, Ahuja N, Jair KW, Li Q, Ohe-Toyota M, Baylin SB, Issa JP: Identification of differentially methylated sequences in colorectal cancer by methylated CpG island amplification. Cancer Res 1999, 59:2307-2312.

43. Yan PS, Chen CM, Shi H, Rahmatpanah F, Wei SH, Huang TH: Applications of CpG island microarrays for high-throughput analysis of DNA methylation. J Nutr 2002, I 32:2430S-2434S.

44. Chen CM, Chen HL, Hsiau TH, Hsiau AH, Shi H, Brock GJ, Wei SH, Caldwell CW, Yan PS, Huang TH: Methylation target array for rapid analysis of CpG island hypermethylation in multiple tissue genomes. Am J Pathol 2003, I 63:37-45. 
45. Song F, Smith JF, Kimura MT, Morrow AD, Matsuyama T, Nagase H, Held WA: Association of tissue-specific differentially methylated regions (TDMs) with differential gene expression. Proc Natl Acad Sci USA 2005, I02:3336-334I.

46. Frigola J, Song J, Stirzaker C, Hinshelwood RA, Peinado MA, Clark SJ: Epigenetic remodeling in colorectal cancer results in coordinate gene suppression across an entire chromosome band. Nat Genet 2006, 38:540-549.

47. Shen L, Kondo Y, Guo Y, Zhang J, Zhang L, Ahmed S, Shu J, Chen X, Waterland RA, Issa JP: Genome-wide profiling of DNA methylation reveals a class of normally methylated CpG island promoters. PLoS Genet 2007, 3:2023-2036.

48. Sakamoto H, Suzuki M, Abe T, Hosoyama T, Himeno E, Tanaka S, Greally JM, Hattori N, Yagi S, Shiota K: Cell type-specific methylation profiles occurring disproportionately in CpG-less regions that delineate developmental similarity. Genes Cells 2007, I 2: I | 23-1| 32

49. Down TA, Rakyan VK, Turner DJ, Flicek P, Li H, Kulesha E, Graf S, Johnson N, Herrero J, Tomazou EM, Thorne NP, Backdahl L, Herberth M, Howe KL, Jackson DK, Miretti MM, Marioni JC, Birney E, Hubbard TJ, Durbin R, Tavare S, Beck S: A Bayesian deconvolution strategy for immunoprecipitation-based DNA methylome analysis. Nat Biotechnol 2008, 26:779-785.

50. Rauch TA, Wu X, Zhong X, Riggs AD, Pfeifer GP: A human B cell methylome at 100-base pair resolution. Proc Natl Acad Sci USA 2009, 106:67|-678.

5I. Beck S, Rakyan VK: The methylome: approaches for global DNA methylation profiling. Trends Genet 2008, 24:23 I-237.

52. Shiota K, Kogo Y, Ohgane J, Imamura T, Urano A, Nishino K, Tanaka $\mathrm{S}$, Hattori N: Epigenetic marks by DNA methylation specific to stem, germ and somatic cells in mice. Genes Cells 2002, 7:961-969

53. Tompa R, McCallum CM, Delrow J, Henikoff JG, van Steensel B, Henikoff S: Genome-wide profiling of DNA methylation reveals transposon targets of CHROMOMETHYLASE3. Curr Biol 2002, I 2:65-68.

54. Tran RK, Henikoff JG, Zilberman D, Ditt RF, Jacobsen SE, Henikoff S: DNA methylation profiling identifies CG methylation clusters in Arabidopsis genes. Curr Biol 2005, 15:154-159.

55. Buckley PG, Jarbo C, Menzel U, Mathiesen T, Scott C, Gregory SG, Langford CF, Dumanski JP: Comprehensive DNA copy number profiling of meningioma using a chromosome I tiling path microarray identifies novel candidate tumor suppressor loci. Cancer Res 2005, 65:2653-2661.

56. RepeatMasker Open-3.0 [http://www.repeatmasker.org]

57. Feinberg AP, Vogelstein B: A technique for radiolabeling DNA restriction endonuclease fragments to high specific activity. Anal Biochem 1983, 132:6-13.

58. Ge X, Yamamoto S, Tsutsumi S, Midorikawa $Y$, Ihara S, Wang SM, Aburatani $\mathrm{H}$ : Interpreting expression profiles of cancers by genome-wide survey of breadth of expression in normal tissues. Genomics 2005, 86: 127-I4I.

59. Laitinen PJ, Brown KM, Piippo K, Swan H, Devaney JM, Brahmbhatt B, Donarum EA, Marino M, Tiso N, Viitasalo M, Toivonen L, Stephan $\mathrm{DA}$, Kontula $\mathrm{K}$ : Mutations of the cardiac ryanodine receptor (RyR2) gene in familial polymorphic ventricular tachycardia. Circulation 2001, 103:485-490.

60. Wehrens XH, Lehnart SE, Huang F, Vest JA, Reiken SR, Mohler PJ, Sun J, Guatimosim S, Song LS, Rosemblit N, D'Armiento JM, Napolitano C, Memmi M, Priori SG, Lederer WJ, Marks AR: FKBPI2.6 deficiency and defective calcium release channel (ryanodine receptor) function linked to exercise-induced sudden cardiac death. Cell 2003, I I 3:829-840.

61. Morita H, Seidman J, Seidman CE: Genetic causes of human heart failure. J Clin Invest 2005, I I 5:5 I8-526.

62. Wu X, Chang B, Blair NS, Sargent M, York AJ, Robbins J, Shull GE, Molkentin JD: Plasma membrane Ca2+-ATPase isoform 4 antagonizes cardiac hypertrophy in association with calcineurin inhibition in rodents. J Clin Invest 2009, I 1 9:976-985.

63. Ladd-Acosta C, Pevsner J, Sabunciyan S, Yolken RH, Webster MJ, Dinkins T, Callinan PA, Fan JB, Potash JB, Feinberg AP: DNA methylation signatures within the human brain. Am J Hum Genet 2007, 8 I:|304-13|5

64. Illingworth $R$, Kerr A, Desousa D, Jorgensen $H$, Ellis $P$, Stalker J, Jackson D, Clee C, Plumb R, Rogers J, Humphray S, Cox T, Langford C,
Bird A: A novel CpG island set identifies tissue-specific methylation at developmental gene loci. PLoS Biol 2008, 6:e22.

65. Oakes CC, La Salle S, Smiraglia DJ, Robaire B, Trasler JM: A unique configuration of genome-wide DNA methylation patterns in the testis. Proc Natl Acad Sci USA 2007, 104:228-233.

66. Kapranov P, Cheng J, Dike S, Nix DA, Duttagupta R, Willingham AT, Stadler PF, Hertel J, Hackermuller J, Hofacker IL, Bell I, Cheung E, Drenkow J, Dumais E, Patel S, Helt G, Ganesh M, Ghosh S, Piccolboni A, Sementchenko V, Tammana H, Gingeras TR: RNA maps reveal new RNA classes and a possible function for pervasive transcription. Science 2007, 3 16: | 484- I 488.

67. Branco MR, Pombo A: Intermingling of chromosome territories in interphase suggests role in translocations and transcription-dependent associations. PLOS Biol 2006, 4:e I 38

68. Zilberman D, Gehring M, Tran RK, Ballinger T, Henikoff S: Genomewide analysis of Arabidopsis thaliana DNA methylation uncovers an interdependence between methylation and transcription. Nat Genet 2007, 39:6I-69.

69. Cooperative Human Tissue Network [http://chtn.nci.nih.gov]

70. Fiegler H, Carr P, Douglas EJ, Burford DC, Hunt S, Scott CE, Smith J, Vetrie D, Gorman P, Tomlinson IP, Carter NP: DNA microarrays for comparative genomic hybridization based on DOP-PCR amplification of BAC and PAC clones. Genes Chromosomes Cancer 2003, 36:36I-374.

7I. Cleveland WS, Grosse E, Shyu WM: Local Regression Models. In Statistical Models Edited by: Chambers JM, Hastie TJ. Pacific Grove, CA: S. Wadsworth \& Brooks/Cole; 1992.

72. Locke DP, Sharp AJ, McCarroll SA, McGrath SD, Newman TL, Cheng Z, Schwartz S, Albertson DG, Pinkel D, Altshuler DM, Eichler EE: Linkage disequilibrium and heritability of copy-number polymorphisms within duplicated regions of the human genome. Am J Hum Genet 2006, 79:275-290.

73. Ihaka R, Gentleman RR: R: a language for data analysis and graphics. J Comput Graph Stat 1996, 5:299-3।4.

74. UCSC Genome Browser [http://genome.ucsc.edu]

75. Irizarry RA, Hobbs B, Collin F, Beazer-Barclay YD, Antonellis $\mathrm{KJ}$, Scherf U, Speed TP: Exploration, normalization, and summaries of high density oligonucleotide array probe level data. Biostatistics 2003, 4:249-264.

76. SCGAP Hematopoietic Stem Cells Project [http:// www.cbil.upenn.edu/SCGAP/resources.html]

77. Halekoh U, Højsgaard S, Yan J: The R package geepack for generalized estimating equations. J Stat Softw 2005, I5: I-II.

78. Lorincz MC, Schübeler D, Goeke SC, Walters M, Groudine M, Martin $D I$ Dynamic analysis of proviral induction and De Novo methylation: implications for a histone deacetylase-independent, methylation density-dependent mechanism of transcriptional repression. Mol Cell Biol 2000, 20:842-850.

79. Tusnady GE, Simon I, Varadi A, Aranyi T: BiSearch: primer-design and search tool for PCR on bisulfite-treated genomes. Nucleic Acids Res 2005, 33:e9.

80. Ewing B, Green P: Base-calling of automated sequencer traces using phred. II. Error probabilities. Genome Res 1998, 8: $186-194$

8I. Ewing B, Hillier L, WendI MC, Green P: Base-calling of automated sequencer traces using phred. I. Accuracy assessment. Genome Res 1998, 8: 175-185.

82. Gordon D, Abajian C, Green P: Consed: a graphical tool for sequence finishing. Genome Res 1998, 8:195-202. 